\title{
PROJECTIVE DIFFERENTIAL GEOMETRY OF DEVELOPABLE
}

\section{SURFACES}

BY

\section{WILLIAM WELLS DENTON}

$\S 1$. The simultaneous solutions of an involutory system of two linear homogeneous partial differential equations of the second order, with two independent variables, and a similar equation of the third order.

Let there be given a system of partial differential equations of the form

$$
A y_{u u}+B y_{u v}+C y_{v v}+D y_{u}+E y_{v}+F y=0
$$

where

$$
A^{\prime} y_{u u}+B^{\prime} y_{u v}+C^{\prime} y_{v v}+D^{\prime} y_{u}+E^{\prime} y_{v}+F^{\prime} y=0 \text {, }
$$

$$
y_{u}=\frac{\partial y}{\partial u}, \quad y_{v}=\frac{\partial y}{\partial v}, \quad y_{u v}=\frac{\partial^{2} y}{\partial u \partial v}, \quad \text { etc. }
$$

and where $A, B, \cdots F^{\prime}$ are analytic functions of $u$ and $v$, such that not all of the second order determinants of the matrix

vanish.

$$
\left\|\begin{array}{ccc}
A & B & C \\
A^{\prime} & B^{\prime} & C^{\prime}
\end{array}\right\|
$$

Differentiation of these equations with respect to $u$ and $v$ gives

$$
\begin{gathered}
A y_{u u u}+B y_{u u v}+C y_{u v v}+{ }^{*}+\left(A_{u}+D\right) y_{u u}+\left(B_{u}+E\right) y_{u v} \\
+C_{u} y_{v v}+\left(D_{u}+F\right) y_{u}+E_{u} y_{v}+F_{u} y=0, \\
*+A y_{u u v}+B y_{u v v}+C y_{v v v}+A_{v} y_{u u}+\left(B_{v}+D\right) y_{u v} \\
+\left(C_{v}+E\right) y_{v v}+D_{v} y_{u}+\left(E_{v}+F\right) y_{v}+F_{v} y=0, \\
A^{\prime} y_{u u u}+B^{\prime} y_{u u v}+C^{\prime} y_{u v v}+{ }^{*}+\left(A_{u}^{\prime}+D^{\prime}\right) y_{u u} \\
+\left(B_{u}^{\prime}+E^{\prime}\right) y_{u v}+C_{u}^{\prime} y_{v v}+\left(D_{u}^{\prime}+F^{\prime}\right) y_{u}+E_{u}^{\prime} y_{v}+F_{u}^{\prime} y=0, \\
+A^{\prime} y_{u u v}+B^{\prime} y_{u v v}+C^{\prime} y_{v v v}+A^{\prime} y_{u u}+\left(B_{v}^{\prime}+D^{\prime}\right) y_{u v} \\
+\left(C_{v}^{\prime}+E^{\prime}\right) y_{v v}+D_{v}^{\prime} y_{u}+\left(E_{v}^{\prime}+F^{\prime}\right) y_{v}+F_{v}^{\prime} y=0,
\end{gathered}
$$

so that it is possible to express the third, and indeed all higher derivatives of

* Presented to the Society (Chicago), April 6, 1912. 
$y$ in the form

$$
\alpha y_{u u}+\beta y_{u v}+\gamma y_{v v}+\delta y_{u}+\epsilon y_{v}+\zeta y,
$$

provided the determinant of the coefficients,

$$
\left|\begin{array}{llll}
A & B & C & 0 \\
0 & A & B & C \\
A^{\prime} & B^{\prime} & C^{\prime} & 0 \\
0 & A^{\prime} & B^{\prime} & C^{\prime}
\end{array}\right|=K,
$$

is not identically equal to zero.

The theory of the invariants and covariants of system (1), in the case that $K$ does not vanish identically, forms a basis for the projective differential geometry of non-developable surfaces. That theory has been developed by Professor Wilczynski.* The object of this paper is to give a similar theory for developable surfaces. The writer takes this opportunity to thank Professor Wilczynski for personally directing this work.

We shall consider a system of form (1) for which $K$ is identically equal to zero: Since not all of the second order determinants of the matrix (2) vanish, all of the third order minors of $K$ cannot be identically equal to zero. Therefore, if, in addition to equations (1), any linear homogeneous partial differential equation of the third order,

$$
\begin{aligned}
G y_{u u u}+ & H y_{u v v}+I y_{u v v}+J y_{v v v v} \\
& +A^{\prime \prime} y_{u u}+B^{\prime \prime} y_{u v}+C^{\prime \prime} y_{v v}+D^{\prime \prime} y_{u}+E^{\prime \prime} y_{v}+F^{\prime \prime} y=0,
\end{aligned}
$$

independent of equations (3) be given, it will again be possible to express all the derivatives of $y$ in the form (4).

The new system, composed of a system of equations of form (1), for which $K$ vanishes identically, and of equation (5), and for which certain integrability conditions, to be discussed presently, are satisfied, will be called system $(A)$. The theory of the invariants and covariants of this system forms a basis for the projective differential geometry of developable surfaces, as will be shown.

Let $y$ be a solution of system $(A)$, analytic in the vicinity of $\left(u_{0}, v_{0}\right)$, so that it may be written

$$
\begin{aligned}
y=y^{0}+y_{u}^{0}\left(u-u_{0}\right) & +y_{v}^{0}\left(v-v_{0}\right)+\frac{1}{2}\left[y_{u u}^{0}\left(u-u_{0}\right)^{2}\right. \\
& \left.+2 y_{u v}^{0}\left(u-u_{0}\right)\left(v-v_{0}\right)+y_{v v}\left(v-v^{0}\right)^{2}\right]+\cdots,
\end{aligned}
$$

*E. J. Wurczrnsin, Projective Differential Geometry of Curved Surfaces. See especially Fourth memoir, these Transactions, vol. 10 (1909), p. 76. See also preceding memoirs, vol. 8 (1907), p. 233; vol. 9 (1908), p. 79; p. 293.

t Unless otherwise stated, the term developable surfaces will be understood to include cones. 
where $y^{0}, y_{u}^{0}$, etc., are the values of $y, y_{u}, \cdots$, for $u=u_{0}, v=v_{0}$. Since all derivatives of $y$ can be expressed uniquely in the form (4), the series (6) may be put in the form

$$
y=y^{0} y^{\prime}+y_{u}^{0} y^{\prime \prime}+y_{v}^{0} y^{(3)}+y_{u u}^{0} y^{(4)}+y_{u v}^{0} y^{(5)}+y_{v v}^{0} y^{(6)},
$$

where $y^{\prime}, y^{\prime \prime}, \cdots y^{(6)}$ are analytic functions of $u-u_{0}, v-v_{0}$. The number of linearly independent analytic solutions of $(A)$ is equal to the number of arbitrary constants in the general solution (7). On account of equations (1) not all of the six constants, $y_{0}, y_{u}^{0}, \cdots y_{v v}^{0}$, are arbitrary. The following system of equations is always equivalent to system (1),

$$
\begin{aligned}
*+(A B) y_{u v}+(A C) y_{v v}+(A D) y_{u}+(A E) y_{v}+(A F) y & =0, \\
(8) \quad(B A) y_{u u}+{ }^{*}+(B C) y_{v v}+(B D) y_{u}+(B E) y_{v}+(B F) y & =0, \\
(C A) y_{u u}+(C B) y_{u v}+{ }^{*}+(C D) y_{u}+(C E) y_{v}+(C F) y & =0,
\end{aligned}
$$

where $(A B)=A B^{\prime}-A^{\prime} B$, etc. The following cases may be distinguished.

I. If the determinants $(A B),(B C),(C A)$ are all different from zero, each of the second derivatives of $y$ may be expressed linearly in terms of any one of them and $y, y_{u}, y_{v}$; so that every expression of form (4) may be reduced to any one of the following forms

$$
\begin{aligned}
& \alpha_{1} y_{u u}+\beta_{1} y_{u}+\gamma_{1} y_{v}+\delta_{1} y, \\
& \alpha_{2} y_{u v}+\beta_{2} y_{u}+\gamma_{2} y_{v}+\delta_{2} y, \\
& \alpha_{3} y_{v v}+\beta_{3} y_{u}+\gamma_{3} y_{v}+\delta_{3} y .
\end{aligned}
$$

Since $K=(C A)^{2}-(A B)(B C)$ is identically equal to zero, if one of the three determinants $(A B),(B C),(C A)$ vanishes, at least one of the others must also be equal to zero. We therefore consider the following additional possibilities.

II. If $(A B)$ and $(B C)$ were identically equal to zero, $(C A)$ would also be identically equal to zero, and the above equations (8) would reduce to the first order or disappear. Both possibilities have been excluded by the assumption that the second order determinants of the matrix (2) are not all equal to zero.

III. If $(B C)$ and $(C A)$ are identically equal to zero, while $(A B)$ is different from zero, every expression of form (4) may be reduced to form (11).

IV. If $(C A)$ and $(A B)$ are identically equal to zero, while $(B C)$ is different from zero, every expression of form (4) may be reduced to form (9).

It is to be noticed that forms (9) and (11) are identical, except for an interchange of the variables $u$ and $v$, so that cases III and IV may be regarded as identical except for the notation. 
Multiplying equations (3), in order, by the minors of the first, second, third and fourth elements in the first column of $K$, and adding, we find

$$
A_{1} y_{u u}+B_{1} y_{u v}+C_{1} y_{v v}+D_{1} y_{u}+E_{1} y_{v}+F_{1} y=0 \text {, }
$$

where

$$
A_{1}=\left|\begin{array}{cccc}
A_{u}+D & B & C & 0 \\
A v & A & B & C \\
A_{u}+D^{\prime} & B^{\prime} & C^{\prime} & 0 \\
A_{v} & A^{\prime} & B^{\prime} & C^{\prime}
\end{array}\right|, \text { etc. }
$$

Let the left member of equation (12) be reduced by means of equations (8) to one of the above forms, (9), (10), or (11), so that (12) takes the form

$$
\alpha w+\beta y_{u}+\gamma y_{v}+\delta y=0,
$$

where $w$ stands for $y_{u u}, y_{u v}$, or $y_{v v}$. If all four of the coefficients of the new equation are not identically equal to zero, it is possible, by means of this equation, to reduce any expression of the chosen form to one involving not more than three terms. In this case system $(A)$ cannot have more than three linearly independent analytic solutions. If all of the coefficients of equation (13) are identically equal to zero, equations (1) are said to be in involution. In this case there is a possibility that the four quantities $y, y_{u}, y_{v}, w$ remain arbitrary, so that system $(A)$ has four and only four linearly independent solutions.

It must be noticed, however, that some of the derivatives of $y$ may be computed in more than one way; for example

$$
y_{u v v v}=\frac{\partial y_{u v v}}{\partial v}=\frac{\partial y_{v v v}}{\partial u} \text {. }
$$

In order, therefore, that the expressions of form (4) for the derivatives may be consistent, certain so-called integrability conditions must be satisfied. They are of the form (13). Let there be $n$ independent conditions in the set, from which all others may be derived.

$$
\alpha_{k} w+\beta_{k} y_{u}+\gamma_{k} y_{v}+\delta_{k} y=0 \quad(k=1,2, \cdots, n) .
$$

The number of linearly independent quantities, $w, y_{u}, y_{v}, y$, is reduced by at least one for each non-identically satisfied equation of the set (14). But if equations (14) are all identically satisfied, that is, if

$$
\alpha_{k}=\beta_{k}=\gamma_{k}=\delta_{k}=0 \quad(k=1,2, \cdots, n),
$$

all four quantities, $w, y_{u}, y_{v}, y$, may remain arbitrary, so that system $(A)$ has four linearly independent solutions. 
The following theorem may now be stated. Given a system of partial differential equations of form (A), consisting of a system of form (1), for which $K$ is identically equal to zero, and an equation of form (5), not derivable from equations (1), all of whose coefficients are analytic functions. ( $\alpha$ ) If the determinants $(A B),(B C),(C A)$ are not all identically equal to zero, $(\beta)$ if further the equations (1) are in involution, $(\gamma)$ if finally the integrability conditions are identically satisfied, system $(A)$ has four and only four linearly independent analytic solutions. If any one of the above conditions $(\alpha),(\beta),(\gamma)$, is not satisfied, the number of linearly independent solutions of system $(A)$ is less than, or exceeds. four.

To complete the proof of this theorem it suffices to apply well-known existence theorems.

§2. Geometric interpretation. The integrating developable surface.

If the conditions $(\alpha),(\beta),(\gamma)$ are satisfied, system $(A)$ has precisely four linearly independent analytic solutions. Let these be

$$
y^{(k)}=f^{(k)}(u, v) \quad(k=1,2,3,4),
$$

Interpret $y^{(k)}$ as the homogeneous coördinates of a point $y$ in space. As $u$ and $v$ assume all of their values, $y$ describes a surface $S$, an integral surface of the system. This surface cannot degenerate into a curve. For if $S$ degenerated in that way, the ratios of $y^{(1)}, y^{(2)}, y^{(3)}, y^{(4)}$ would be functions of a single variable $t=\tau(u, v)$, and $y^{(1)}, y^{(2)}, y^{(3)}, y^{(4)}$, themselves would be solutions of some linear partial differential equations of the first order,* and this is contrary to the assumption that the system has four linearly independent solutions.

The surface $S$ is not unique. The most general system of linearly independent analytic solutions of $(A)$ is

$$
\eta_{i}=\sum_{k=1}^{4} c_{i k} y_{k} \quad(i=1,2,3,4),
$$

where the determinant of the constant coefficients $c_{i k}$ does not vanish. The most general integral surface of $(A)$ is, therefore, a projective transformation of any particular one.

The integral surface is, moreover, a developable. This follows from the fact that equations (1) are in involution. Conversely, it can be shown that every developable is an integral surface of an involutory system of form (1). A proof of these statements has been given by Professor Wilczynski. $\dagger$

Therefore, the theory of a system of partial differential equations of form $(A)$, for which the conditions $(\alpha),(\beta),(\gamma)$ are all satisfied, is identical with the projective differential geometry of developable surfaces in three-dimensional space.

- Ibid., vol. 8, p. 237.

† Loc. cit., pp. 238-240. 
§3. First intermediate form. The surface referred to its generators.

For present purpose, it will be convenient to take system $(A)$ in the following form

$$
\begin{aligned}
y_{u u} & =a y_{v v}+b y_{u}+c y_{v}+d y, \\
y_{u v} & =a^{\prime} y_{v v}+b^{\prime} y_{u}+c^{\prime} y_{v}+d^{\prime} y, \\
y_{v v v} & =a^{\prime \prime} y_{v v}+b^{\prime \prime} y_{u}+c^{\prime \prime} y_{v}+d^{\prime \prime} y,
\end{aligned}
$$

which will hereafter be called system $(B)$. It was shown in $\S 1$ that system ( $A$ ) can always be reduced to the form $(B)$, except that if $(C A)$ and $(A B)$ are identically equal to zero, the variables $u$ and $v$ must be interchanged. The theory, therefore, can suffer no essential loss of generality in consequence of this choice of the form $(B)$.

For system $(B)$, the value of $K$ is $a-{a^{\prime}}^{2}$, and this is, of course, assumed to be identically equal to zero.

Differentiation of equations (16) and (17), with respect to $u$ and $v$, gives

$$
\begin{aligned}
y_{u u u}-a y_{u v v} & =\alpha_{1} y_{v v}+\beta_{1} y_{u}+\gamma_{1} y_{v}+\delta_{1} y, \\
y_{u u v}-a^{\prime} y_{u v v} & =\alpha_{2} y_{v v}+\beta_{2} y_{u}+\gamma_{2} y_{v}+\delta_{2} y, \\
y_{u u v}-a y_{v v v} & =\alpha_{3} y_{v v}+\beta_{3} y_{u}+\gamma_{3} y_{v}+\delta_{3} y, \\
y_{u v v}-a^{\prime} y_{v v v} & =\alpha_{4} y_{v v}+\beta_{4} y_{u}+\gamma_{4} y_{v}+\delta_{4} y,
\end{aligned}
$$

where $\alpha_{1}=a_{u}+a b+c a^{\prime}$, etc.

Multiplying equation (20) by $a^{\prime}$, (21) by $-a^{\prime}$, (22) by $a$, and adding, we find

$$
\begin{aligned}
\left(a-a^{\prime 2}\right) y_{u v v}= & \left(a^{\prime} \alpha_{2}-a^{\prime} \alpha_{3}+a \alpha_{4}\right) y_{v v}+\left(a^{\prime} \beta_{2}-a^{\prime} \beta_{3}+a \beta_{4}\right) y_{u} . \\
& +\left(a^{\prime} \gamma_{2}-a^{\prime} \gamma_{3}+a \gamma_{4}\right) y_{v}+\left(a^{\prime} \delta_{2}-a^{\prime} \delta_{3}+a \delta_{4}\right) y .
\end{aligned}
$$

Since equations (16) and (17) are in involution, all of the coefficients in equation (23) must be identically equal to zero.

It will not be necessary to develop the integrability conditions explicitly at the present moment. It is assumed that they are satisfied identically, so that system $(B)$ has four and only four linearly independent analytic solutions.

If a surface is represented by equations of the form (15), a transformation of the form

$$
\bar{u}=\phi(u, v), \quad \bar{v}=\psi(u, v),
$$

will modify at most the parametric curves on the surface but not the surface itself.

Let the transformation (24) be applied to system $(B)$. If the resulting system be solved for $\bar{y}_{u u}$ and $\bar{y}_{u v}$, the new coefficients corresponding to $a$ 
and $a^{\prime}$ are found to be

$$
\bar{a}=\frac{\psi_{u}^{2}-2 a^{\prime} \psi_{u} \psi_{v}+a \psi_{v}^{2}}{\phi_{v}^{2}-2 a^{\prime} \phi_{u} \phi_{v}+a \phi_{v}^{2}}, \quad \bar{a}^{\prime}=\frac{\phi_{u} \psi_{u}+a \phi_{v} \psi_{v}-a^{\prime} \phi_{u} \psi_{v}-a^{\prime} \phi_{v} \psi_{u}}{\phi_{u}^{z}-2 a^{\prime} \phi_{u} \phi_{v}+a \phi_{v}^{2}},
$$

which give $\bar{a}-\bar{a}^{\prime 2}=0$, as they should. The other coefficients of the new equations of the second order are fractions with the common denominator

for example,

$$
\Delta=\left(\phi_{u} \psi_{v}-\phi_{v} \psi_{u}\right)\left(\phi_{u}^{2}-2 a^{\prime} \phi_{u} \phi_{v}+a \phi_{v}^{2}\right) ;
$$

$$
\begin{aligned}
\Delta \bar{c}=( & \left.-\psi_{u u}+a \psi_{v v}+a^{\prime} b \psi_{v}+c \psi_{v}\right)\left(\phi_{u} \psi_{v}+a^{\prime} \psi_{v} \phi_{v}-2 a^{\prime} \phi_{v} \psi_{v}\right) \\
& -2\left(-a \phi_{v} \psi_{v}+a^{\prime} \phi_{u} \psi_{v}\right)\left(-\psi_{u v}+a^{\prime} \psi_{v v}+a^{\prime} b^{\prime} \psi_{v}+c^{\prime} \psi_{v}\right) .
\end{aligned}
$$

The coefficients of the new equation of the third order, obtained by solving the transforms of equations (18), (19), (21), (22) for $\bar{y}_{v v v}$ are fractions with the common denominator $\left(\phi_{u} \psi_{v}-\phi_{v} \psi_{u}\right)^{6}$.

If $\psi$ be chosen so as to satisfy the equation

$$
\psi_{u} \psi_{v}^{-1}=a^{\prime},
$$

the numerators of $\bar{a}$ and $\bar{a}^{\prime}$ will both vanish. The denominators,

$$
\phi_{u}^{2}-2 a^{\prime} \phi_{u} \phi_{v}+a \phi_{v}^{2}=\left(\phi_{u}-a^{\prime} \phi_{v}\right)^{2},
$$

however, cannot vanish; for, if this expression were equal to zero, it would be necessary to have

$$
\phi_{u} \phi_{v}^{-1}=a^{\prime}=\psi_{u} \psi_{v}^{-1}, \text { or } \phi_{u} \psi_{v}-\phi_{v} \psi_{u}=0 \text {, }
$$

contrary to the assumption that $\phi$ and $\psi$ are independent functions of $u$ and $\boldsymbol{v}$. If, therefore, the transformation be subjected to the single condition (27), the new equations of the second order will be free from the terms in $\bar{y}_{\boldsymbol{v}}$.

If $\bar{a}=\bar{a}^{\prime}=0$, the original system has the desired form without transformation. Suppose this to be the case, or imagine that a first transformation of this character has been made. Then the system to be considered has the form

$$
\begin{aligned}
y_{u u} & =b y_{u}+c y_{v}+d y, \\
y_{u v} & =b^{\prime} y_{u}+c^{\prime} y_{v}+d^{\prime} y, \\
y_{v v v} & =a^{\prime \prime} y_{v v}+b^{\prime \prime} y_{u}+c^{\prime \prime} y_{v}+d^{\prime \prime} y .
\end{aligned}
$$

This will be called system (C), or the first intermediate form of system $(B)$.

Equation (23) shows that the two equations of the second order, which appear in this system, are always in involution. It is not necessary, therefore, from now on, to pay attention to the involutorial conditions. 
The integrability conditions for this new system require that $c$ be equal to zero identically. This is seen at once, by differentiating equation (28) with respect to $v$, (29) with respect to $u$, and equating the coefficients of $y_{v v}$ in the resulting expressions for $y_{u u v}$.

Since $c$ is zero, equation (28) shows that the curves $v=$ const., upon the integral surfaces of system $(C)$, are straight lines. The reduction of a system of form $(A)$ to form $(C)$ is, therefore, equivalent to the determination of the straight line generators of its developable integral surfaces.

\$4. Second intermediate form. The surface referred to its plane sections. Equations (25) and (26) show that the form of system $(C)$ is not changed by any transformation of the form

$$
\bar{u}=\phi(u, v), \quad \bar{v}=\psi(v),
$$

where $\phi$ and $\psi$ are arbitrary functions of the arguments indicated. Making this transformation, and solving the resulting equations for $\bar{y}_{v v v}$, we find for the new coefficient corresponding to $b^{\prime \prime}$ in the original system,

$$
\overline{b^{\prime \prime}}=\phi_{u} \psi_{v}^{-3}\left(b^{\prime \prime}+\chi\right) \text {, }
$$

where

$$
\begin{aligned}
\chi= & \theta\left(c^{\prime \prime}+2 a^{\prime \prime} b^{\prime}-3 b_{v}-3 b^{\prime 2}\right) \\
& +\theta^{2}\left(2 a^{\prime \prime} c^{\prime}-a^{\prime \prime} b-9 b^{\prime} c^{\prime}+3 b_{v}+3 b b^{\prime}-3 c_{v}^{\prime}-3 d^{\prime}\right) \\
& +\theta^{3}\left(3 b c^{\prime}-6 c^{\prime 2}-3 b^{2}-3 b_{u}-3 d\right) \\
& +\theta^{2} \eta\left(a^{\prime \prime}+6 c^{\prime}-6 b\right)+a^{\prime \prime} \theta^{2} \eta^{2}-12 c^{\prime} \theta^{3} \eta \\
& +9 \theta^{3} \eta^{2}-\theta_{v v}+2 \theta \theta_{u v}+a^{\prime \prime} \theta_{u v}+\theta_{u} \theta_{v}-\theta \theta_{u}^{2}+a^{\prime \prime} \theta_{u}^{2} \\
& +\theta \theta_{v}\left(-6 c^{\prime}-3 b\right)-3 b^{\prime} \theta_{v}+3 b^{\prime} \theta \theta_{u}-9 b \theta^{2} \theta_{u}+6 \theta \theta_{v} \eta \\
& +10 \theta^{2} \theta_{u} \eta+2 a^{\prime \prime} \theta \theta_{u} \eta+a^{\prime \prime} \theta_{v} \eta+\theta^{3} \eta_{u}+5 \theta^{2} \eta_{v}+a^{\prime \prime} \theta \eta_{v}, \\
& \theta=\phi_{v} \phi_{u}^{-1}, \quad \eta=\phi_{u u} \phi_{u}^{-1} .
\end{aligned}
$$

It is possible, therefore, to make $\bar{b}^{\prime \prime}=0$, by solving a differential equation of the third order for the function $\phi$, viz.,

$$
b^{\prime \prime}+x=0 \text {. }
$$

Imagine this to have been done, or that $b^{\prime \prime}$ is already equal to zero. Then the system of equations under discussion has the following form,

$$
\begin{aligned}
& y_{u u}=b y_{u}+d y, \\
& y_{u v}=b^{\prime} y_{u}+c^{\prime} y_{v}+d^{\prime} y, \\
& y_{v v v}=a^{\prime \prime} y_{v v}+c^{\prime \prime} y_{v}+d^{\prime \prime} y,
\end{aligned}
$$


which will be called form $(D)$, or the second intermediate form of system $(B)$.

Equation (36) shows that the curves $u=$ const., upon the integral surfaces of system $(D)$, are plane curves. Conversely, if the curves $u=$ const. are plane curves, the four linearly independent analytic functions, $y^{(1)}, y^{(2)}$, $y^{(3)}, y^{(4)}$, must satisfy an equation of this form. Therefore, the reduction of system $(C)$ to a system of form $(D)$, or the reduction of system $(A)$ to a system which contains an equation of form (36), is equivalent to the determination of a oneparameter family of plane sections of the integral developable surfaces of the original system.

Whenever the generators and a one parameter family of plane sections of a developable are given there may be constructed immediately a system of form $(D)$ satisfied by the developable. If, however, a general system of form $(A)$ be given, so that the finite equations of its generators and plane sections are unknown, their determination with reference to the known elements requires the integrations involved in reducing the given system of form $(A)$ to the second intermediate form.

§5. The integrability conditions for a system in the second intermediate form. The canonical form.

It will be necessary to develop explicitly the integrability conditions for system $(D)$. These are obtained by putting

$$
\frac{\partial y_{u u}}{\partial v}=\frac{\partial y_{u v}}{\partial u}, \quad \frac{\partial y_{v v v}}{\partial u}=\frac{\partial^{2} y_{u v}}{\partial v^{2}} .
$$

From equations (34), (35), (36), we find by differentiation,

$$
\begin{aligned}
y_{u u v}=\left(b_{v}^{\prime}+b b^{\prime}\right) y_{u}+\left(d+b c^{\prime}\right) y_{v}+\left(d_{v}+b d^{\prime}\right) y, \\
y_{u v u}=\left(b_{u}^{\prime}+b b^{\prime}+c^{\prime} b^{\prime}+d^{\prime}\right) y_{u}+\left(c_{u}^{\prime}+c^{\prime 2}\right) y_{v}+\left(b^{\prime} d+c^{\prime} d^{\prime}+d_{u}^{\prime}\right) y, \\
y_{v v v u}=\left(a_{u}^{\prime \prime}+c^{\prime} a^{\prime \prime}\right) y_{v v}+\left[a^{\prime \prime}\left(b_{v}^{\prime}+b^{\prime 2}\right)+b^{\prime} c^{\prime \prime}+d^{\prime \prime}\right] y_{u} \\
+\left[a^{\prime \prime}\left(c_{v}^{\prime}+d^{\prime}+b^{\prime} c^{\prime}\right)+c_{u}^{\prime \prime}+c^{\prime} c^{\prime \prime}\right] y_{v} \\
+\left[a^{\prime \prime}\left(d_{v}^{\prime}+b^{\prime} d^{\prime}\right)+c^{\prime \prime} d^{\prime}+d_{u}^{\prime \prime}\right] y, \\
y_{u v v v}=\left(2 c_{v}^{\prime}+d^{\prime}+b^{\prime} c^{\prime}+a^{\prime \prime} c^{\prime}\right) y_{v v}+\left[\left(b_{v}^{\prime}+b^{\prime 2}\right)_{v}+b^{\prime}\left(b_{v}^{\prime}+b^{\prime 2}\right)\right] y_{u} \\
+\left[c^{\prime}\left(b_{v}^{\prime}+b^{\prime 2}\right)+\left(c_{v}^{\prime}+d^{\prime}+b^{\prime} c^{\prime}\right)_{v}+d_{v}^{\prime}+b^{\prime} d^{\prime}+c^{\prime} c^{\prime \prime}\right] y_{v} \\
+\left[d^{\prime}\left(b_{v}^{\prime}+b^{\prime 2}\right)+\left(d_{v}^{\prime}+b^{\prime} d^{\prime}\right)_{v}+c^{\prime} d^{\prime \prime}\right] y .
\end{aligned}
$$

Equations (37) ensure unique values for all third and fourth order derivatives. That the expressions for all higher order derivatives will also be unique then follows directly by differentiation. Since these equations must hold for all 
values of $y_{v v}, y_{u}, y_{v}, y$, it is necessary to have,

$$
\begin{gathered}
b_{v}=b_{u}^{\prime}+b^{\prime} c^{\prime}+d^{\prime}, \\
d+b c^{\prime}=c_{u}^{\prime}+c^{\prime 2}, \\
b d^{\prime}+d_{v}=d_{u}^{\prime}+c^{\prime} d^{\prime}+b^{\prime} d, \\
a_{u}^{\prime \prime}=2 c_{v}^{\prime}+d^{\prime}+b^{\prime} c^{\prime}, \\
a^{\prime \prime}\left(b_{v}^{\prime}+b^{\prime 2}\right)+b^{\prime} c^{\prime \prime}+d^{\prime \prime}=\left(b_{v}^{\prime}+b^{\prime 2}\right)_{v}+b^{\prime}\left(b_{v}^{\prime}+b^{\prime 2}\right), \\
a^{\prime \prime}\left(b^{\prime} c^{\prime}+d^{\prime}+c_{v}^{\prime}\right)-\left(b^{\prime} c^{\prime}+d^{\prime}+c_{v}^{\prime}\right)_{v}-\left(b^{\prime} d^{\prime}+d_{v}^{\prime}\right)+c_{v}^{\prime \prime} \\
\left.a^{\prime \prime}\left(b^{\prime} d^{\prime}+d_{v}^{\prime}\right)+c^{\prime \prime} d^{\prime}+b^{\prime 2}\right)=0,
\end{gathered}
$$

The first and fourth of these equations give

$$
\left(a^{\prime \prime}+b^{\prime}\right)_{u}=\left(2 c^{\prime}+b\right)_{v} \text {. }
$$

It is, therefore, possible to find a function $p$, of $u$ and $v$, satisfying the following conditions,

$$
p_{u}=2 c^{\prime}+b, \quad p_{v}=a^{\prime \prime}+b^{\prime} .
$$

Transform system $(D)$ by putting

$$
y=\lambda \bar{y},
$$

where $\lambda$ is an arbitrary function of $u$ and $v$. Since the coördinates are homogeneous, the integral surface of the system will remain unchanged under this transformation. The form of the system, moreover, remains unchanged, and the coefficients of the new equations are found to be

$$
\begin{aligned}
\bar{b} & =b-2 \lambda_{u} \lambda^{-1}, \\
\bar{d} & =d+b \lambda_{u} \lambda^{-1}-\lambda_{u u} \lambda^{-1}, \\
\overline{b^{\prime}} & =b^{\prime}-\lambda_{v} \lambda^{-1}, \\
\bar{c}^{\prime} & =c^{\prime}-\lambda_{u} \lambda^{-1}, \\
\bar{d}^{\prime} & =d^{\prime}+c^{\prime} \lambda_{v} \lambda^{-1}-\lambda_{u v} \lambda^{-1}+b^{\prime} \lambda_{u} \lambda^{-1}, \\
\bar{a}^{\prime \prime} & =a^{\prime \prime}-3 \lambda_{v} \lambda^{-1}, \\
\bar{c}^{\prime \prime} & =c^{\prime \prime}+2 a^{\prime \prime} \lambda_{v} \lambda^{-1}-3 \lambda_{v v} \lambda^{-1}, \\
\bar{d}^{\prime \prime} & =d^{\prime \prime}+a^{\prime \prime} \lambda_{v v} \lambda^{-1}+c^{\prime \prime} \lambda_{v} \lambda^{-1}-\lambda_{v v v} \lambda^{-1} .
\end{aligned}
$$

The expressions corresponding to $p_{u}$ and $p_{v}$ for the new equations are (43) $\bar{p}_{u}=2 \bar{c}^{\prime}+\bar{b}=2 c^{\prime}+b-4 \lambda_{u} \lambda^{-1}, \bar{p}_{v}=a^{\prime \prime}+\bar{b}^{\prime}=a^{\prime \prime}+b^{\prime}-4 \lambda_{v} \lambda^{-1}$, 
so that they may be made to vanish by putting

$$
y=\lambda \bar{y}, \quad \lambda=e^{p / 4} .
$$

Let this restriction be placed upon the transformation (41) which has just been applied to system $(D)$. Then the new coefficients, which will be denoted by German letters, are found, by specializing equations (42), to be

$$
\begin{array}{rlrl}
\mathfrak{b} & =b-2 e, & \mathfrak{b}^{\prime} & =d^{\prime}+c^{\prime} e^{\prime}-e_{v}-e e^{\prime}+b^{\prime} e, \\
\mathfrak{b} & =d+b e-e_{u}-e^{2}, & \mathfrak{a}^{\prime \prime} & =a^{\prime \prime}-3 e^{\prime}, \\
\mathfrak{b}^{\prime} & =b^{\prime}-e^{\prime}, & \mathfrak{c}^{\prime \prime} & =c^{\prime \prime}+2 a^{\prime \prime} e^{\prime}-3 e_{v}^{\prime}-3 e^{2}, \\
\mathfrak{c}^{\prime} & =c^{\prime}-e, & \mathfrak{b}^{\prime \prime} & =d^{\prime \prime}+a^{\prime \prime}\left(e_{v}^{\prime}+e^{\prime 2}\right)+c^{\prime \prime} e^{\prime}-e_{v v}^{\prime} \\
& -e^{3}-3 e^{\prime} e_{v}^{\prime},
\end{array}
$$

where

and, of course,

$$
4 e^{\prime}=a^{\prime \prime}+b^{\prime}, \quad 4 e=2 c^{\prime}+b,
$$

$$
\mathfrak{b}=-2 \mathfrak{c}^{\prime}, \quad \mathfrak{a}^{\prime \prime}=-\mathfrak{b}^{\prime} .
$$

The new system, which will be called $(E)$, has the canonical form

$$
\begin{aligned}
y_{u u} & =-2 \mathfrak{c}^{\prime} y_{u}+\delta y, \\
y_{u v} & =\mathfrak{b}^{\prime} y_{u}+\mathfrak{c}^{\prime} y_{v}+\mathfrak{b}^{\prime} y, \\
y_{v v v} & =-\mathfrak{b}^{\prime} y_{v v}+\mathfrak{c}^{\prime \prime} y_{v}+\mathfrak{b}^{\prime \prime} y .
\end{aligned}
$$

Any system of form $(A)$ can be reduced to such a canonical form. The integrability conditions for system $(E)$ may be found from equations (38). They are as follows:

$$
\begin{aligned}
& -2 c_{v}^{\prime}=\mathfrak{b}_{w}^{\prime}+\mathfrak{b}^{\prime} \mathfrak{c}^{\prime}+\mathfrak{b}^{\prime}, \\
& \delta=c_{u}^{\prime}+3 c^{\prime 2} \text {, } \\
& \delta_{v}=\delta_{u}^{\prime}+3 c^{\prime} \delta^{\prime}+\mathfrak{b}^{\prime} \delta \text {, } \\
& \mathfrak{b}^{\prime} \mathfrak{c}^{\prime \prime}+\mathfrak{b}^{\prime \prime}=\mathfrak{b}_{v v}^{\prime}+4 \mathfrak{b}^{\prime} \mathfrak{b}_{v}^{\prime}+2 \mathfrak{b}^{\prime 3} \text {, } \\
& \mathfrak{c}_{u}^{\prime \prime}=\mathfrak{c}_{v v}^{\prime}+2\left(\mathfrak{b}^{\prime} \mathfrak{c}^{\prime}+b^{\prime}\right)_{v}+2\left(\mathfrak{b}^{\prime} \mathfrak{c}^{\prime}+b^{\prime}\right) \mathfrak{b}^{\prime}, \\
& \delta_{w}^{\prime \prime}=\delta_{v 0}^{\prime}+2\left(b^{\prime} \delta^{\prime}\right)_{v}+2 \mathfrak{b}^{\prime 2} \delta^{\prime}+c^{\prime \prime} \delta^{\prime}-c^{\prime} \delta^{\prime \prime} \text {. }
\end{aligned}
$$

\$6. Seminvariants and semi-covariants.

Let the system of differential equations $(D)$ be transformed by putting

$$
y=\lambda y,
$$

where $\lambda$ is an arbitrary function of $u$ and $v$. The result of the transformation 
is given by equations (42). Those functions of the coefficients of $(D)$, of $y$, and of the derivatives of these quantities, which are left unchanged by every such transformation, will be called seminvariants or semi-covariants, according as they do not, or do, contain $y$ and its derivatives. It is easily verified that each of the coefficients (45) of the canonical form $(E)$ is a seminvariant, also that the derivatives of these eight quantities are seminvariants. Every seminvariant of $(D)$ is a function of the six quantities $b, \mathfrak{b}^{\prime}, \mathfrak{c}^{\prime}, \mathfrak{b}^{\prime}, \mathfrak{c}^{\prime \prime}, \mathfrak{b}^{\prime \prime}$, and of their derivatives. For, in the first place, $\mathfrak{b}$ and $\mathfrak{a}^{\prime \prime}$ are expressible in terms of $\mathfrak{c}^{\prime}$ and $\mathfrak{b}^{\prime}$ respectively, and, in the second place, every equation of form $(D)$ can be reduced to form $(E)$ by a transformation of form (44). But the value of any seminvariant of $(D)$ is the same as for any system, e. g. $(E)$, obtained from $(D)$ by a transformation of form (44), and it therefore reduces to a function of the coefficients of $(E)$.

Since all of the derivatives of $y$ may be expressed in terms of the four quantities $y, y_{u}, y_{v}, y_{v v}$, it is necessary to find only such semi-covariants as involve these four quantities.

The transformation gives

$$
\begin{array}{rlrl}
\bar{y} & =\lambda^{-1} y, & \bar{y}_{u} & =\lambda^{-1}\left(y_{u}-\lambda_{u} \lambda^{-1} y\right), \\
\bar{y}_{v} & =\lambda^{-1}\left(y_{v}-\lambda_{v} \lambda^{-1}\right) y, & \bar{y}_{v v} & =\lambda^{-1}\left[y_{v v}-2 \lambda_{v} \lambda^{-1} y_{v}\right. \\
& & \left.+\left(2 \lambda_{v}^{2} \lambda^{-2}-\lambda_{v v} \lambda^{-1}\right) y\right],
\end{array}
$$

while equations (43) give

$$
\bar{e}=e-\lambda_{u} \lambda^{-1}, \quad \bar{e}^{\prime}=e^{\prime}-\lambda_{v} \lambda^{-1} ;
$$

whence may be found the following relative semi-covariants

$$
\begin{gathered}
y, \quad \mathfrak{q}=y_{u}-e y, \quad \mathfrak{r}=y_{v}-e^{\prime} y, \\
\mathfrak{B}=y_{v v}-2 e^{\prime} y_{v}+\left(e^{\prime 2}-e_{v}^{\prime}\right) y=\mathfrak{r}_{v}-e^{\prime} \mathfrak{r},
\end{gathered}
$$

which satisfy the equations

$$
\bar{y}=\lambda^{-1} y, \quad \overline{\mathfrak{q}}=\lambda^{-1} \mathfrak{q}, \quad \overline{\mathfrak{r}}=\lambda^{-1} \mathfrak{r}, \quad \overline{\mathrm{s}}=\lambda^{-1} \mathfrak{b} .
$$

\section{§ 7. Invariants and covariants.}

Equation (32) shows that form $(D)$ is left unchanged by any transformation of the form $\bar{u}=\phi(u, v), \bar{v}=\psi(v)$, where $\psi$ is an arbitrary function of $v$, and $\phi$ is any function of $u$ and $v$, which satisfies the differential equation (33). In particular, it will be convenient to make the transformation

$$
\bar{u}=\phi(u), \quad \bar{v}=\psi(v),
$$

where $\phi$ and $\psi$ are arbitrary functions of the single arguments indicated. It will therefore be assumed that a definite family of plane sections of the 
integral surface has been chosen, and that this family is to be preserved in the transformation.

The coefficients of the new equations, obtained by applying the transformation (58) to system $(D)$ are

$$
\begin{aligned}
& \bar{b}^{\prime}=\phi_{u}^{-1}(b-\eta), \quad \bar{d}=\phi_{u}^{-2} d, \\
& \bar{b}^{\prime}=\psi_{v}^{-1} b^{\prime}, \quad \bar{c}^{\prime}=\phi_{u}^{-1} c^{\prime}, \quad \bar{d}^{\prime}=\phi_{u}^{-1} \psi_{v}^{-1} d^{\prime},
\end{aligned}
$$$$
\bar{a}^{\prime \prime}=\psi_{0}^{-1}\left(a^{\prime \prime}-3 \zeta\right), \quad \bar{c}^{\prime \prime}=\psi_{0}^{-2}\left(c^{\prime \prime}+a^{\prime \prime} \zeta-\zeta_{v}-\zeta^{2}\right), \quad \bar{d}^{\prime \prime}=\psi_{0}^{-3} d^{\prime \prime},
$$
where

$$
\eta=\phi_{u u} \phi_{u}^{-1}, \quad \zeta=\psi_{v v} \psi_{v}^{-1} .
$$

Those functions of the seminvariants and their derivatives which are left unchanged by a transformation of form (58) are called invariants. The seminvariants are all functions of the six quantities $b, \mathfrak{b}^{\prime}, \mathfrak{c}^{\prime}, \mathfrak{b}^{\prime}, \mathfrak{c}^{\prime \prime}, \mathfrak{b}^{\prime \prime}$, and their derivatives. The effect of the transformation (58) upon these quantities must, therefore, be investigated. Equations (45) and (59) give

$$
4 \bar{e}=\phi_{u}^{-1}(4 e-\eta), \quad 4 \bar{e}^{-}=\psi_{0}^{-1}\left(4 e^{\prime}-3 \zeta\right),
$$

and also,

$$
\begin{aligned}
& \overline{\mathfrak{b}}=\phi_{u}^{-2}\left(\mathfrak{b}+\frac{1}{2} \mathfrak{c}^{\prime} \eta+\frac{1}{4} \eta_{u}-\frac{1}{16} \eta^{2}\right), \overline{\mathfrak{b}^{\prime}}=\psi_{v}^{-1}\left(\mathfrak{b}^{\prime}+\frac{3}{4} \zeta\right), \\
& \overline{\mathfrak{b}^{\prime}}=\phi_{u}^{-1} \psi_{v}^{-1}\left(\mathfrak{b}^{\prime}-\frac{3}{4} \mathfrak{c}^{\prime} \zeta-\frac{1}{4} \mathfrak{b}^{\prime} \eta-\frac{3}{16} \eta \zeta\right), \overline{\mathfrak{c}^{\prime}}=\phi_{u}^{-1}\left(\mathfrak{c}^{\prime}+\frac{1}{4} \eta\right), \\
& \overline{\mathfrak{c}^{\prime \prime}}=\psi_{v}^{-2}\left(\mathfrak{c}^{\prime \prime}+\frac{1}{2} \mathfrak{b}^{\prime} \zeta+\frac{6}{4} \zeta_{v}-\frac{7}{16} \zeta^{2}\right), \\
& \overline{\mathfrak{b}^{\prime \prime}}=\psi_{v}^{-3}\left(\mathfrak{b}^{\prime \prime}-\frac{3}{4} \mathfrak{c}^{\prime \prime} \zeta-\frac{9}{16} \mathfrak{b}^{\prime} \zeta^{2}+\frac{3}{4} \mathfrak{b}^{\prime} \zeta_{v}+\frac{2}{6} \frac{7}{4} \zeta^{3}-\frac{2}{16} \zeta \zeta_{v}+\frac{3}{4} \zeta_{v v}\right), \\
& \overline{\mathfrak{b}_{v}^{\prime}}=\psi_{v}^{-2}\left(\mathfrak{b}_{v}^{\prime}-\mathfrak{b}^{\prime} \zeta+\frac{3}{4} \zeta_{v}-\frac{3}{4} \zeta^{2}\right) .
\end{aligned}
$$

A sum will be said to be isobaric if, in this transformation, each of its terms is multiplied by the same product of the form $\phi_{u}^{\lambda}$ or $\psi_{v}^{\mu}$. Every invariant must be isobaric.

From equations (61), the following relative invariants are found directly

$$
\mathfrak{D}^{\prime}=\mathfrak{b}^{\prime}+\mathfrak{b}^{\prime} \mathfrak{c}^{\prime}, \quad \mathfrak{C}^{\prime \prime}=\mathfrak{c}^{\prime \prime}-\frac{18}{9} \mathfrak{b}^{\prime 2}-\frac{\mathfrak{b}}{3} \mathfrak{b}_{v}^{\prime} .
$$

If $\mathfrak{D}^{\prime}$ is different from zero, the further relative invariants

$$
\mathfrak{P}^{\prime}=\mathfrak{b}^{\prime}+\frac{3}{4} \frac{\mathfrak{D}_{\mathfrak{v}}^{\prime}}{\mathfrak{D}^{\prime}}, \quad \mathbb{C}^{\prime}=\mathfrak{c}^{\prime}+\frac{1}{4} \frac{\mathfrak{D}_{u}^{\prime}}{\mathfrak{D}^{\prime}},
$$

may be obtained. In fact, these four quantities satisfy the equations

$$
\overline{\mathfrak{B}^{\prime}}=\psi_{v}^{-1} \mathfrak{B}^{\prime}, \quad \overline{\mathfrak{C}}^{\prime}=\phi_{u}^{-1} \mathfrak{C}^{\prime}, \quad \overline{\mathfrak{D}^{\prime}}=\phi_{u}^{-1} \psi_{v}^{-1} \mathfrak{D}^{\prime}, \quad \overline{\mathfrak{C}}^{\prime \prime}=\psi_{v}^{-2} \mathfrak{C}^{\prime \prime} .
$$

The seminvariants (45) of system $(D)$ satisfy the integrability conditions of system (E). Equations (50) and (52) together with (62) and (63) show 
that it is possible to express the six seminvariants $b, \mathfrak{b}^{\prime}, \mathfrak{c}^{\prime}, \mathfrak{b}^{\prime}, \mathfrak{c}^{\prime \prime}, \mathfrak{b}^{\prime \prime}$, and their derivatives in terms of the four invariants $\mathfrak{B}^{\prime}, \mathbb{E}^{\prime}, \mathfrak{D}^{\prime}, \mathfrak{C}^{\prime \prime}$, and their derivatives. Equations (49), (51), (53), (54) show, moreover, that these four invariants and their derivatives are not independent. In fact, the first three equations give respectively

$$
\begin{aligned}
& 8 \mathfrak{C}_{v}^{\prime}+4 \mathfrak{D}^{\prime}+4 \mathfrak{P}_{u}^{\prime}-5 \frac{\partial^{2}}{\partial u \partial v} \log \dot{\mathfrak{D}}^{\prime}=0,
\end{aligned}
$$

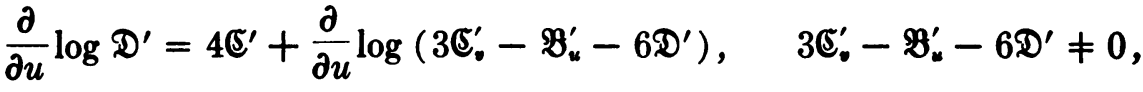

$$
\begin{aligned}
& 4 \mathfrak{P}^{\prime}\left(13 \mathfrak{P}_{u}^{\prime}-39 \mathfrak{C}_{v}^{\prime}-42 \mathfrak{D}^{\prime}\right)-3\left(13 \mathfrak{P}_{u}^{\prime}-39 \mathfrak{C}_{v}^{\prime}-42 \mathfrak{D}\right) \frac{\partial}{\partial v} \log \mathfrak{D}^{\prime} \\
& +45 \mathfrak{C}_{u}^{\prime \prime}-45 \mathfrak{C}_{v v}^{\prime}+75 \mathfrak{P}_{u v}^{\prime}-72 \mathfrak{E}_{v}^{\prime}-36 \mathfrak{D}^{\prime}-36 \mathfrak{P}_{u}^{\prime}-90 \mathfrak{D}_{v}^{\prime}=0 .
\end{aligned}
$$

The condition that the expression $3 \mathfrak{C}_{v}^{\prime}-\mathfrak{B}_{u}^{\prime}-6 \mathfrak{D}^{\prime}$ shall not vanish, requires, as will be seen, only that the integral surface be not a cone.

Let it be assumed that $\mathbb{C}^{\prime \prime}$ is different from zero, and put

so that

$$
\mathbb{E}=\frac{\mathfrak{D}^{\prime 2}}{\mathbb{C}^{\prime \prime}},
$$

$$
\bar{E}=\phi_{u}^{-2} \text { E. }
$$

Let it also be assumed that $\mathfrak{O}^{\prime}$ and $\mathfrak{C}^{\prime}$ are different from zero, and introduce two symbols of operation

It is clear that

$$
U=\frac{1}{\mathfrak{C}^{\prime}} \frac{\partial}{\partial u}, \quad V=\frac{1}{\mathfrak{B}^{\prime}} \frac{\partial}{\partial v} .
$$

$$
U\left(\mathfrak{O}^{\prime}\right), \quad V\left(\mathbb{E}^{\prime}\right), \quad U\left(\mathbb{E}^{\prime \prime}\right), \quad V(\mathbb{E}),
$$

are invariants. In fact, they satisfy the following equations,

$$
\begin{aligned}
& \bar{U}\left(\overline{\mathscr{P}}^{\prime}\right)=\psi_{0}^{-1} U\left(\mathbb{C}^{\prime}\right), \quad \bar{V}\left(\overline{\mathbb{S}}^{\prime}\right)=\phi_{u}^{-1} V\left(\mathbb{\mho}^{\prime}\right), \quad \bar{U}\left(\overline{\mathbb{S}}^{\prime \prime}\right)=\psi_{0}^{-2} U\left(\mathbb{E}^{\prime \prime}\right), \\
& \bar{V}(\bar{\Xi})=\phi_{u}^{-2} V(\mathbb{E}) \text {. }
\end{aligned}
$$

From any two invariants $\lambda$ and $\mu$ for which $\bar{\lambda}=\phi_{u}^{-l} \lambda, \bar{\mu}=\phi_{u}^{-m} \mu$, it is always possible to form a new invariant

$$
\left(\mu, \lambda_{u}\right)=m \mu \lambda_{u}-\lambda \mu_{u},
$$

their Wronskian with respect to $u$, which satisfies the equation

$$
\left(\bar{\mu}, \bar{\lambda}_{u}\right)=\phi_{u}^{-l-m}\left(\mu, \lambda_{u}\right) \text {. }
$$

By repeating and combining the Wronskian process and the operations $U$ and $V$, an infinite number of invariants can be deduced from $\mathfrak{O}^{\prime}, \mathbb{C}^{\prime}, \mathbb{C}^{\prime \prime}$, and $\mathbb{E}$. It can now be shown that all invariants are functions of those ob- 
tained in this way. It has been seen that the seminvariants (45) and their derivatives may be expressed in terms of $\mathfrak{O}^{\prime}, \mathbb{E}^{\prime}, \mathbb{V}^{\prime \prime}$, and $\mathfrak{D}^{\prime}$. It has also been seen that all invariants are functions of the seminvariants (45) and their derivatives. Therefore any invariant may be expressed as a function of $\mathfrak{O}^{\prime}, \mathbb{C}^{\prime}, \mathbb{C}^{\prime \prime}, \mathbb{E}$, and derivatives.

Let the invariants be divided into two classes, of $\phi$ - and $\psi$-invariants, according as their transforms are multiplied by powers of $\phi_{u}$ or $\psi_{v}$ respectively. By a process of induction precisely parallel to that employed by Professor Wilczynski, for a similar system of invariants, ${ }^{*}$ it can be shown that there are $n^{2}+2 n+2$ independent (except for equations (54) and (64)) $\phi$-invariants of orders equal to or less than $n$, and the same number of $\psi$-invariants of the orders named; moreover, that the invariants may be chosen so as to contain all the derivatives of $\mathfrak{O}^{\prime}, \mathbb{E}^{\prime}, \mathbb{E}^{\prime \prime}, \mathbb{E}$, up to, and including, those of order $n$; and, finally, that the two classes of $\phi$-invariants and $\psi$-invariants are independent of each other. If equations (54) and (64) are taken into account, the number of invariants of orders equal to or less than $n$ must be reduced by $\frac{1}{2}\left(3 n^{2}+3 n-4\right)$. The $W$ ronskian, the $U$-process, and the V-process suffice, therefore, to deduce all invariants from the four fundamental ones $\mathfrak{B}^{\prime}, \mathfrak{C}^{\prime}$, $\mathbb{S}^{\prime \prime}, \mathbb{E}$. The number of independent invariants of order equal to or less than $n$ is $\frac{1}{2}\left(n^{2}+5 n+12\right)$, for $n \geq 2$. For $n=1$ there are eight independent invariants.

Applying the transformation (58) to the semi-covariants (57), we find

$$
\begin{gathered}
\bar{y}=y, \quad \overline{\mathfrak{q}}=\phi_{\bar{v}}^{-1}\left(\mathfrak{q}+\frac{1}{4} \eta y\right), \quad \overline{\mathfrak{r}}=\psi_{v}^{-1}\left(\mathfrak{r}+\frac{3}{4} \zeta y\right), \\
=\psi_{v}^{-2}\left[\bar{z}+\frac{1}{2} \mathfrak{r} \zeta+\frac{1}{4}\left(\zeta^{2}+3 \zeta_{v}\right) y\right] ;
\end{gathered}
$$

whence we obtain the following relative covariants,

$$
\begin{gathered}
y, \quad \mathbb{Q}=\mathfrak{q}-\mathfrak{c}^{\prime} y, \quad \Re=\mathfrak{r}-\mathfrak{b}^{\prime} y, \\
\mathbb{S}=\mathbb{Q}-\frac{2}{3} b^{\prime} \mathfrak{r}+\frac{1}{8}\left(4 \mathfrak{b}^{\prime 2}-9 \mathfrak{b}_{\mathfrak{v}}^{\prime}\right) y .
\end{gathered}
$$

They satisfy the equations

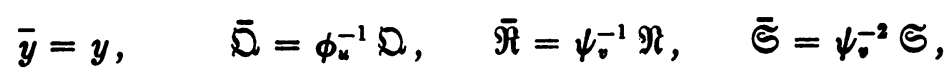

and are clearly independent. All covariants may be expressed in terms of the covariants (66) and the invariants.

8 8. Principle of duality. The adjoint system, and its integral curve.

Given a system of partial differential equations in the second intermediate form $(D)$, for which the conditions $(\alpha),(\beta),(\gamma)$ are satisfied, so that it has

- Loc. cit., pp. 254, 255. 
four linearly independent analytic solutions

$$
y^{(k)}=f^{(k)}(u, v) \quad(k=1,2,3,4),
$$

which may be interpreted as the homogeneous coördinates of a point $y$ of a developable surface $S$. The equation of the plane $\lambda$, tangent to $S$ at $y$, is

$$
\left|\begin{array}{llll}
x_{1} & x_{2} & x_{3} & x_{4} \\
y^{(1)} & y^{(2)} & y^{(3)} & y^{(4)} \\
y_{u}^{(1)} & y_{u}^{(2)} & y_{u}^{(3)} & y_{u}^{(4)} \\
y_{v}^{(1)} & y_{v}^{(2)} & y_{v}^{(3)} & y_{v}^{(4)}
\end{array}\right|=0,
$$

so that the coördinates of $\lambda$ are proportional to the minors of $x_{1}, x_{2}, x_{3}, x_{4}$, each of which may be represented by an expression of the form

$$
\lambda=\left|y, y_{u}, y_{v}\right|,
$$

which stands for a determinant of the third order of the matrix

$$
\left\|y^{(k)}, y_{u}^{(k)}, y_{v}^{(k)}\right\|
$$

Equation (68), together with (34), (35) and (36), gives

$$
\lambda_{u}=\left(b+c^{\prime}\right) \lambda \text {, }
$$

from which it is possible, by differentiation, to find expressions for all the derivatives of $\lambda$ taken at least once with respect to $u$, as $\lambda_{u u}, \lambda_{u v}, \lambda_{u u v}, \lambda_{u v v}$, etc. The following equations hold for the derivatives of $\lambda$ taken with respect to $v$ alone

$$
\begin{gathered}
-\lambda_{v}+b^{\prime} \lambda+\left|y, y_{u}, y_{v v}\right|=0, \\
\left(\lambda_{v v}\right)+4 e^{\prime}\left|y, y_{u}, y_{v v}\right|+\left|y_{v}, y_{u}, y_{v v}\right|+c^{\prime}\left|y, y_{v}, y_{v v}\right|=0, \\
\left(\lambda_{v v v}\right)+4\left(e_{v}^{\prime}+4{e^{\prime}}^{2}\right)\left|y, y_{u}, y_{v v}\right|+8 e^{\prime}\left|y_{v}, y_{u}, y_{v v}\right| \\
+\left(8 e^{\prime} c^{\prime}-L\right)\left|y, y_{v}, y_{v v}\right|=0, \\
\left(\lambda_{v v v v}\right)+f\left|y, y_{u}, y_{v v}\right|+g\left|y_{v}, y_{u}, y_{v v}\right|+h\left|y, y_{v}, y_{v v}\right|=0,
\end{gathered}
$$

where

$$
\begin{gathered}
\left(\lambda_{v v}\right)=-\lambda_{v v}+b^{\prime} \lambda_{v}+\left(b_{v}^{\prime}+c^{\prime \prime}\right) \lambda, \\
\left(\lambda_{v v v}\right)=-\lambda_{v v v}+b^{\prime} \lambda_{v v}+\left(2 b_{v}^{\prime}+c^{\prime \prime}\right) \lambda_{v}+\left(b_{v v}^{\prime}+c_{v}^{\prime \prime}+4 e^{\prime} c^{\prime \prime}-d^{\prime \prime}\right) \lambda, \\
\left(\lambda_{v v v v}\right)=-\lambda_{v v v v}+b^{\prime} \lambda_{v v v}+\left(3 b_{v}^{\prime}+c^{\prime \prime}\right) \lambda_{v v}+\left(3 b_{v v}^{\prime}+2 c_{v}^{\prime \prime}+4 e^{\prime} c^{\prime \prime}-d^{\prime \prime}\right) \lambda_{v} \\
\quad+\left[\left(b_{v v}^{\prime}+c_{v}^{\prime \prime}+4 e^{\prime} c^{\prime \prime}-d^{\prime \prime}\right)_{v}+4 c^{\prime \prime}\left(e_{v}^{\prime}+4 e^{\prime^{2}}\right)-8 e^{\prime} d^{\prime \prime}\right] \lambda, \\
f=4\left(e_{v}^{\prime}+4 e^{\prime^{2}}\right)_{v}+16 e^{\prime}\left(e_{v}^{\prime}+4 e^{\prime 2}\right), \quad g=+12 e_{v}^{\prime}+48 e^{2}, \\
h=4 e^{\prime}\left(4 e^{\prime} c^{\prime}-2 d^{\prime}\right)+4 c^{\prime} e_{v}^{\prime}+\left(8 e^{\prime} c^{\prime}-L\right)_{v}+a^{\prime \prime}\left(8 e^{\prime} c^{\prime}-L\right) .
\end{gathered}
$$


If the invariant expression

$$
L=b^{\prime} c^{\prime}+d^{\prime}-c_{v}^{\prime}=b^{\prime} \mathfrak{c}^{\prime}+\mathfrak{b}^{\prime}-\mathfrak{c}_{v}^{\prime}=-\frac{1}{5}\left(3 \mathfrak{C}_{n}^{\prime}-\mathfrak{B}_{u}^{\prime}-6 \mathfrak{D}^{\prime}\right)
$$

is different from zero, the determinants $\left|y, y_{u}, y_{v v}\right|,\left|y_{v}, y_{u}, y_{v v}\right|,\left|y, y_{v}, y_{v v}\right|$, may be eliminated from the above equations so as to obtain an equation of the fourth order for the coördinates of the plane $\lambda$,

$$
\left|\begin{array}{cccc}
-\lambda_{v}+b^{\prime} \lambda & 1 & 0 & 0 \\
\left(\lambda_{v v}\right) & 4 e^{\prime} & 1 & c^{\prime} \\
\left(\lambda_{v v v}\right) & 4\left(e_{v}^{\prime}+4{e^{\prime 2}}^{2}\right) & 8 e^{\prime} & \delta e^{\prime} c^{\prime}-L \\
\left(\lambda_{v v v v}\right) & f & g & h
\end{array}\right|=0
$$

say, for brevity,

$$
L \lambda_{v v v v}+L_{1} \lambda_{v v v}+L_{2} \lambda_{v v}+L_{3} \lambda_{v}+L_{4} \lambda=0 .
$$

The completely integrable system, composed of equations (69) and (71), which may be called the adjoint system of $(D)$, represents the same integral surface as system $(D)$, but in plane- instead of point-coördinates. The planes whose coördinates are $\lambda^{(1)}, \lambda^{(2)}, \lambda^{(3)}, \lambda^{(4)}$ are the tangent planes of the surface, which, in general, change with $v$. As $u$ changes, each plane remains fixed. Equation (71) may be thought of as the differential equation, in plane coördinates, of the edge of regression, $C$, of the integral developable surface $S$ of system $(D)$. As $v$ changes, the plane $\lambda$ envelops the curve $C$. As $u$ changes, the plane remains fixed, corresponding to the fact that at all points of one and the same generator the developable has the same tangent plane.

If $I$ is identically zero, $\left|y, y_{u}, y_{v v}\right|,\left|y_{v}, y_{u}, y_{v v}\right|,\left|y, y_{v}, y_{v v}\right|$ may be eliminated from the first three of the equations (70), so as to obtain an equation of the third order for the coördinates of the plane $\lambda$,

$$
\begin{aligned}
\lambda_{v v v}+\lambda_{v v} & \left(-8 e^{\prime}-b^{\prime}\right)+\lambda_{v}\left(8 b^{\prime} e^{\prime}+16{e^{\prime}}^{2}-4 e_{v}^{\prime}-2 b_{v}^{\prime}-c^{\prime \prime}\right) \\
& +\lambda\left(4 e^{\prime} c^{\prime \prime}+8 e^{\prime} b_{v}^{\prime}+4 e_{v}^{\prime} b^{\prime}-16 b^{\prime} e^{\prime 2}-b_{v v}^{\prime}-c_{v}^{\prime \prime}+d^{\prime \prime}\right)=0 .
\end{aligned}
$$

In this case, equations (69) and (72) show that the developable degenerates into a cone, since the coördinates of its tangent planes must satisfy a linear homogeneous relation with constant coefficients. In fact, equation (72) shows that $\lambda^{(1)}, \lambda^{(2)}, \lambda^{(3)}, \lambda^{(4)}$ satisfy a relation of the form

$$
\phi_{1}(u) \lambda^{(1)}+\phi_{2}(u) \lambda^{(2)}+\phi_{3}(u) \lambda^{(3)}+\phi_{4}(u) \lambda^{(4)}=0 \text {. }
$$

But according to (69), the ratios of $\lambda^{(1)}, \lambda^{(2)}, \lambda^{(3)}, \lambda^{(4)}$ are independent of $u$, so that such a relation would reduce to one with constant coefficients. Consequently all of the tangent planes of the developable have a point in common, that is, the cuspidal edge of the developable degenerates into a 
point, and the developable is necessarily a cone. Therefore if the invariant $L$ vanishes identically the integral surface of system $(D)$ is a cone.

If the original system is in the canonical form, so that $e^{\prime}=0$, the adjoint system has the comparatively simple form

$$
\begin{gathered}
\lambda_{u}+\mathfrak{c}^{\prime} \lambda=0, \\
L \lambda_{v v v v}-L_{v} \lambda_{v v v}+\left[\mathfrak{b}^{\prime} L_{v}-L\left(3 \mathfrak{b}_{v}^{\prime}+\mathfrak{c}^{\prime \prime}+\mathfrak{b}^{\prime 2}\right)\right] \lambda_{v v} \\
+\left[\left(L_{v}-\mathfrak{b}^{\prime} L\right)\left(2 \mathfrak{b}_{v}^{\prime}+\mathfrak{c}^{\prime \prime}\right)-L\left(3 \mathfrak{b}_{v v}^{\prime}+2 \mathfrak{c}_{v}^{\prime \prime}-\mathfrak{b}^{\prime \prime}\right)\right] \lambda_{v} \\
+\left[\left(L_{v}-\mathfrak{b}^{\prime} L\right)\left(\mathfrak{b}_{v v}^{\prime}+\mathfrak{c}_{v}^{\prime \prime}-\mathfrak{b}^{\prime \prime}\right)-L\left(\mathfrak{b}_{v v v}^{\prime}+\mathfrak{c}_{v v}^{\prime \prime}-\mathfrak{b}_{v}^{\prime \prime}\right)\right] \lambda=0 .
\end{gathered}
$$

\$ 9. The transformation of Laplace. The cuspidal edge of the integral developable.

Consider any surfaces $S_{0}$, not necessarily developable. We shall assume that its homogeneous point-coördinates, $y^{(1)}, y^{(2)}, y^{(3)}, y^{(4)}$, are functions of two parameters, $u$ and $v$, chosen so that the two families of curves, $u=$ const. and $v=$ const., on it, form a conjugate system. DARBOUX has shown* that this condition is fulfilled if, and only.if, the four functions $y^{(k)}$ satisfy an equation of the form

$$
y_{u v}=A(u, v) y_{u}+B(u, v) y_{v}+C(u, v) y \text {. }
$$

The tangents to the family of curves $u=$ const., at the points $y$, form a congruence $\Gamma_{1}$. One sheet of the focal surface of this congruence is the given surface $S_{0}$. The other sheet is the surface $S_{1}$ whose coördinates $y_{1}^{(k)}$ are given by the Laplace transform of $y$ with respect to $v$,

$$
y_{1}=y_{v}-A y \text {. }
$$

Indeed, it is easily verified that $y_{1}$ satisfies an equation of the form

$$
\left(y_{1}\right)_{u}-B y=D y \text {, }
$$

which shows that any line tangent to $S_{0}$ at $y$, along a curve $u=$ const. on $S_{0}$, is also tangent to $S_{1}$ at $y_{1}$, along a curve $v=$ const. on $S_{1}$.

Similarly the tangents to the family of curves $v=$ const., on the given surface $S_{0}$, form a congruence $\Gamma_{-1}$ such that the coördinates of the second sheet $S_{-1}$ of its focal surface are given by the Laplace transform of $y$ with respect to $u$,

$$
y_{-1}=y_{u}-B y \text {. }
$$

The Laplace transformation may be repeated indefinitely, so that, in general, there is obtained an unlimited sequence of surfaces, $S_{k}(k=\cdots-3$, $-2,-1,0,1,2,3, \cdots)$, on each of which the curves $u=$ const. and $v=$ const. are conjugate families; while to each surface $S_{k}$ corresponds a

* Darboux, Leçons sur la theorie génerale des surfaces, vol. I, p. 122. 
pair of congruences $\Gamma_{k+1}, \Gamma_{k-1}$, formed by the tangents to the curves $u=$ const., $v=$ const., respectively.

The case in which one or more of the surfaces $S_{k}$ are developables is of importance for our theory. We have seen that the differential equations of any developable surface may be written in the form $(B)$. The two families of curves, $u=$ const., $v=$ const. are still perfectly arbitrary. They form a conjugate system if, and only if, $a^{\prime}$ vanishes identically. But when $a^{\prime}$ equals zero, system $(B)$ reduces to its first intermediate form $(C)$, and one of the conjugate families necessarily consists of the generators. The form of system (C) shows that this is the family $v=$ const. The other family is left entirely arbitrary. In this case the relation of conjugacy between the two families of curves becomes degenerate.

We now see that the covariant

$$
\mathfrak{L}=y_{u}-c^{\prime} y,
$$

of system $(C)$ is the Laplace transform of $y$ with respect to $u$.

If four linearly independent solutions $y^{(k)}(k=1,2,3,4)$, of system $(C)$ be put for $y$ into this expression, and also into $\mathfrak{D}_{v}$, there will be found two points. The second equation of system $(C)$ gives the following relation between these points,

$$
\mathfrak{D}_{v}=b^{\prime} y_{u}+y\left(d^{\prime}-c_{v}^{\prime}\right) \text {. }
$$

If $u$ is constant, while $v$ varies continuously, $\mathfrak{Q}$ describes, in general, a space curve $u=$ const. $\mathfrak{D}_{v}$ is a point on the tangent to this curve at the point $\mathfrak{Q}$. Equation (75) shows that $\mathfrak{Q}_{v}$ is also a point of the generator (of the surface $S_{0}$ ) containing the points $y, y_{u}, \mathfrak{D}$. The point $\mathfrak{D}$ is, therefore, that point of the generator of the developable $S_{0}$ in which it intersects the cuspidal edge. The ratios of $\mathfrak{Q}^{(1)}, \mathfrak{Q}^{(2)}, \mathfrak{Q}^{(3)}, \mathfrak{Q}^{(4)}$ must, accordingly, be independent of $u$.

The differential equation which characterizes the cuspidal edge of the integral developable $S_{0}$ of system $(C)$, considered as the locus of the point $\mathfrak{D}$ may be found as follows. Multiplying equation (66b) by $b^{\prime}$ and subtracting from equation (75) we find

$$
\mathfrak{D}_{v}-b^{\prime} \mathfrak{D}=L y
$$

where

$$
L=b^{\prime} c^{\prime}+d^{\prime}-c_{v}^{\prime},
$$

is the invariant discussed in $\$ 7$.

If $L$ vanishes identically, equation (76) shows that the ratios of $\mathfrak{Q}^{(k)}$ are independent not only of $u$, but also of $v$, that is, they are constants; and the cuspidal edge is a point, as should be expected, since the integral surface is a cone. 
If $L$ is not identically zero, differentiation of equation (76) gives

$$
\begin{aligned}
\mathfrak{Q}_{v v}-b^{\prime} \mathfrak{Q}_{v}-b_{v}^{\prime} \mathfrak{Q} & =L_{v} y+L y_{v}, \\
\mathfrak{Q}_{v v v}-b^{\prime} \mathfrak{Q}_{v v}-2 b_{v}^{\prime} \mathfrak{Q}_{v}-b_{v v}^{\prime} \mathfrak{Q} & =L_{v v} y+2 L_{v} y_{v}+L y_{v v}, \\
\mathfrak{Q}_{v v v v}-b^{\prime} \mathfrak{Q}_{v v v}-3 b_{v}^{\prime} \mathfrak{Q}_{v v}-3 b_{v v}^{\prime} \mathfrak{Q}_{v}-b_{v v v}^{\prime} \mathfrak{Q}= & \left(L_{v v v}+d^{\prime \prime} L\right) y \\
& +\left(3 L_{v v}+c^{\prime \prime} L\right) y_{v} \\
& +\left(3 L_{v}+a^{\prime \prime} L\right) y_{v v}+b^{\prime \prime} L y_{u} .
\end{aligned}
$$

When $y_{u}$ is replaced by its equal, $\mathfrak{Q}+c^{\prime} y$, the expressions $y, y_{v}, y_{v v}$, may be eliminated from the four equations (76), (77), so that the desired equation is found in the form

$$
E_{0} \mathfrak{Q}_{v v v v}+E_{1} \mathfrak{Q}_{v v v}+E_{2} \mathfrak{Q}_{v v}+E_{3} \mathfrak{L}_{v}+E_{4} \mathfrak{Q}=0 .
$$

The projective properties of the cuspidal edge of the integral surface $S_{0}$ of system $(C)$ may be studied directly from this equation. The projective theory of space curves, considered from this point of view, has already been developed by Professor Wilczynski.* We shall try, therefore, merely to find the relations between the invariants and covariants of this differential equation and the invariants and covariants of system $(C)$.

For this purpose, we shall assume that system $(C)$ has been reduced to its canonical form $(E)$. Its coefficients, then, are seminvariants, which remain unchanged under any transformation of the form

$$
\lambda(u, v) y=\bar{y} .
$$

Therefore the equation corresponding to (78) may be obtained by substituting the expression (76) for $y$ into equation (48) of system $(E)$. This gives for the equation of the cuspidal edge,

$$
\begin{aligned}
{\left[L^{\prime}\left(\mathfrak{Q}_{v}-\mathfrak{b}^{\prime} \mathfrak{Q}\right)\right]_{v v v}=} & -\mathfrak{b}^{\prime}\left[L^{-1}\left(\mathfrak{Q}_{v}-\mathfrak{b}^{\prime} \mathfrak{Q}\right)\right]_{v v} \\
& +\mathfrak{c}^{\prime \prime}\left[L^{-1}\left(\mathfrak{Q}_{v}-\mathfrak{b}^{\prime} \mathfrak{Q}\right)\right]_{v}+\mathfrak{b}^{\prime \prime} L^{-1}\left(\mathfrak{Q}_{v}-\mathfrak{b}^{\prime} \mathfrak{Q}\right),
\end{aligned}
$$

or

$$
\mathfrak{Q}_{v v v v}+4 p_{1} \mathfrak{Q}_{v v v}+6 p_{2} \mathfrak{Q}_{v v}+4 p_{3} \mathfrak{Q}_{v}+p_{4} \mathfrak{Q}=0 \text {, }
$$

where, in particular, $p_{1}=-\frac{3}{4} L_{v} L^{-1}$.

By making the transformation

$$
\mathfrak{Q}=L^{*} \overline{\mathfrak{Q}}
$$

this equation may be reduced to its semi-canonical form

$$
\overline{\mathfrak{D}}_{v v v v}+6 \pi^{(2)} \overline{\mathfrak{Q}}_{v v}+4 \pi^{(3)} \overline{\mathfrak{D}}_{v}+\pi^{(4)} \overline{\mathfrak{L}}=0,
$$

which is characterized by the absence of the term involving the third derivative.

* E. J. Wilczynski, Projective differential geometry of curves and ruled surfaces, Teubner, Leipzig, 1906, Chapter XIII. 
The remaining coefficients will be

where

$$
\begin{aligned}
& \pi^{(2)}=l^{-1}\left[3 l_{v v}+3 m_{v}+\mathfrak{b}^{\prime}\left(2 l_{v}+m\right)-\mathfrak{c}^{\prime \prime} l\right], \\
& \pi^{(3)}=\vdash^{-1}\left[l_{v v v}+3 m_{v v}+\mathfrak{b}^{\prime}\left(l_{v v}+2 m_{v}\right)-\mathfrak{c}^{\prime \prime}\left(l_{v}+m\right)-\mathfrak{b}^{\prime \prime} l\right], \\
& \pi^{(4)}=\vdash^{-1}\left[m_{v v v}+\mathfrak{b}^{\prime} m_{v v}-\mathfrak{c}^{\prime \prime} m_{v}-\mathfrak{d}^{\prime \prime} m\right],
\end{aligned}
$$

$$
l=L^{-1}, \quad m=\frac{3}{4} l^{5} L_{v}-\mathfrak{b}^{\prime} l .
$$

From these expressions for the coefficients a complete system of invariants and covariants may be readily computed. For example, ${ }^{*}$ we have

$$
\theta_{3}=\pi^{(3)}-3 \pi_{v}^{(2)} \text {. }
$$

If $\theta_{3}$ is identically equal to zero, the generators of $S_{0}$ belong to a linear complex. $\dagger$

Consider, next, the covariant

$$
\Re=y_{v}-b^{\prime} y,
$$

of system $(C)$ which was found in $\S 7$. It is the Laplace transform of $y$ with respect to $v$. Therefore, if $y$ is interpreted as a point on the integral surface $S_{0}, \Re$ must be interpreted as a point on the surface $S_{-1}$ which constitutes the second sheet of the congruence $\Gamma_{-1}$ formed by the tangents to the curves $u=$ const. on $S_{0}$. The differential equations for the surface $S_{-1}$ may be found as follows.

The second equation of system $(C)$ enables us to write

$$
\Re_{u}=c^{\prime} y_{v}+\left(d^{\prime}-b_{u}^{\prime}\right) y \text {. }
$$

Multiplying (66c) by $c^{\prime}$ and subtracting from (80), we obtain,

$$
\Re_{u}-c^{\prime} \Re=M y,
$$

where

$$
M=b^{\prime} c^{\prime}+d^{\prime}-b_{u}^{\prime}=4 \mathfrak{D}^{\prime}-2 L=\frac{2}{5}\left(4 \mathfrak{D}^{\prime}+3 \mathfrak{C}_{v}^{\prime}-\mathfrak{B}_{u}^{\prime}\right) .
$$

If the invariant $M$ vanishes identically, equation (81) shows that the surface $S_{-1}$ degenerates into a curve.

If $M$ is not identically equal to zero, the desired equations may be found by eliminating $y, y_{u}, y_{v}, y_{v v}$ from equations $(66 c)$, (81), and others derived from them by differentiation. We thus obtain a system of form (1)

$$
\Re_{u v}=c^{\prime} \Re_{v}+\left(b^{\prime}+\frac{M_{v}}{M}\right) \Re_{u}+\left[M+c_{v}^{\prime}-c^{\prime}\left(b^{\prime}+\frac{M_{v}}{M}\right)\right] \Re,
$$

- Ibid., p. 240.

† Ibid., p. 254. 


$\left|\begin{array}{ccccc}\Re_{v v} & a^{\prime \prime}-b^{\prime} & b^{\prime \prime} & c^{\prime \prime}-2 b_{v}^{\prime} & d^{\prime \prime}-b_{v v}^{\prime} \\ \Re_{v} & 1 & 0 & -b^{\prime} & -b_{v}^{\prime} \\ \Re & 0 & 0 & 1 & -b^{\prime} \\ \Re_{u}-c^{\prime} \Re & 0 & 0 & 0 & M \\ \Re_{u u}-c^{\prime} \Re_{u}-c_{u}^{\prime} \Re & 0 & M & 0 & M_{u}\end{array}\right|=0$,

for which the coefficients of the terms of the second order are

$$
A=C=B^{\prime}=0, \quad B=1, \quad A^{\prime}=b^{\prime \prime} M, \quad C^{\prime}=M^{2},
$$

so that the invariant $K$ has the value $b^{\prime \prime} M^{3}$. The integral surface is developable if, and only if, $b^{\prime \prime}$ vanishes identically.

Therefore, if a one-parameter family of curves, $C$, be drawn upon a developable, the tangents to these curves form, of course, a congruence. The second sheet of the focal surface of this congruence will degenerate into a curve if, and only if, $M$ is equal to zero. If it does not degenerate, it will itself be a developable if, and only if, the curves $C$ are plane curves.

810. Canonical developments of the non-homogeneous coördinates of a developable surface.

The semi-covariants of system $(E)$ were found to be

$$
y, \quad \mathfrak{q}=y_{u}, \quad \mathfrak{r}=y_{v}, \quad \&=y_{v v} .
$$

If four linearly independent solutions, $y^{(1)}, y^{(2)}, y^{(3)}, y^{(4)}$, be substituted for $y$ in these expressions, three points, $q, \mathfrak{r}, \bar{z}$, semi-covariantly related to the point $y$, will be found. These points, together with $y$, form a non-degenerate tetrahedron, except for certain singular points. This tetrahedron will be used as a tetrahedron of reference, and, more specifically, the coördinates of any point with respect to it will be defined in the following way. If we substitute into any expression of the form

$$
\lambda=\alpha \boldsymbol{b}+\beta \mathfrak{q}+\gamma \mathfrak{r}+\delta y,
$$

for $y$ the four linearly independent solutions, $y^{(1)}, y^{(2)}, y^{(8)}, y^{(4)}$, we find four functions, $\lambda^{(1)}, \lambda^{(2)}, \lambda^{(8)}, \lambda^{(1)}$, the homogeneous coördinates of a point $\lambda$. The coördinates of the point $\lambda$ referred to the tetrahedron of reference, $y$, $\mathfrak{q}, \mathfrak{x}, \bar{z}$, are then defined by the equations

$$
x_{1}=\alpha, \quad x_{2}=\beta, \quad x_{3}=\gamma, \quad x_{4}=\delta .
$$

We shall need expressions for the derivatives of $y$ up to and including the 
sixth order. We find, for example,

$$
\begin{aligned}
y_{u} & =\mathfrak{q}, \quad y_{v}=\mathfrak{r}, \\
y_{u u} & =-2 \mathfrak{c}^{\prime} \mathfrak{q}+\mathfrak{b}, \quad y_{u v}=\mathfrak{b}^{\prime} \mathfrak{q}+\mathfrak{c}^{\prime} \mathfrak{x}+\mathfrak{b}^{\prime} y, \quad y_{v v}=\mathfrak{b}, \\
y_{u u u} & =\left(7 \mathfrak{c}^{\prime 2}-\mathfrak{c}_{u}\right) \mathfrak{q}+\left(\mathfrak{b}_{u}-2 \mathfrak{c}^{\prime} \mathfrak{b}\right) y, \\
y_{u u v} & =-2\left(\mathfrak{c}_{v}^{\prime}+\mathfrak{c}^{\prime} \mathfrak{b}^{\prime}\right) \mathfrak{q}+\left(\mathfrak{b}-2 \mathfrak{c}^{\prime 2}\right) \mathfrak{r}+\left(\mathfrak{b}_{v}-2 \mathfrak{c}^{\prime} \mathfrak{b}^{\prime}\right) y, \\
y_{u v v} & =\mathfrak{c}^{\prime}+\left(\mathfrak{b}_{v}^{\prime}+\mathfrak{b}^{\prime 2}\right) \mathfrak{q}+\left(\mathfrak{c}_{v}^{\prime}+\mathfrak{b}^{\prime}+\mathfrak{b}^{\prime} \mathfrak{c}^{\prime}\right) \mathfrak{r}+\left(\mathfrak{b}_{v}^{\prime}+\mathfrak{b}^{\prime} \mathfrak{b}^{\prime}\right) y, \\
y_{v v v} & =-\mathfrak{b}^{\prime}+\mathfrak{c}^{\prime \prime} \mathfrak{q}+\mathfrak{b}^{\prime \prime} y .
\end{aligned}
$$

All such expressions are of the form

$$
y_{u_{i} v_{j}}=A^{i j} \dot{j}+B^{i j} \mathfrak{q}+C^{i j} \mathfrak{r}+D^{i j} y,
$$

where the subscript $u_{i} v_{j}$ indicates $i+j$ differentiations, $i$ with respect to $u$ and $j$ with respect to $v$. The explicit expressions for the coefficients $A^{i j}$, $B^{i j}, \cdots$, will be omitted for $i+j_{i}>3$, since they are rather long, and since their calculation is of little interest and offers no difficulty.

We shall assume that the point $y$ of the surface corresponds to the value $(0,0)$ of $u$ and $v$, an assumption which involves no loss of generality. If the surface is analytic in the vicinity of this point, for values of $u$ and $v$ sufficiently small, the general solution of system $(E)$ may be expanded into a power series

$$
Y=y+y_{u} u+y_{v} v+\frac{1}{2}\left(y_{u u} y^{2}+2 y_{u v} u v+y_{v v} v^{2}\right)+\cdots,
$$

which may be written in the form

$$
Y=t_{1} \mathfrak{s}+t_{2} \mathfrak{q}+t_{3} \mathfrak{x}+t_{4} y .
$$

The coefficients, $t_{1}, t_{2}, t_{3}, t_{4}$, are power series, which, in accordance with our convention, represent the coördinates of any point of the surface, within a certain vicinity of the point $y$, referred to the tretahedron $y, \mathfrak{q}, \mathfrak{x}, \mathfrak{c}$. These series are

$$
\begin{aligned}
& t_{1}=\frac{1}{2} v^{2}+\frac{1}{2} \mathfrak{c}^{\prime} u v^{2}-\frac{1}{6} \mathfrak{b}^{\prime} v^{3}+\frac{1}{4 !} A^{40} u^{4}+\cdots, \\
& \begin{aligned}
t_{2}=u-\mathfrak{c}^{\prime} u^{2}+\mathfrak{b}^{\prime} u v+\frac{1}{6}\left(7 \mathfrak{c}^{\prime 2}-\mathfrak{c}_{*}^{\prime}\right) u^{3}-\left(\mathfrak{c}_{0}^{\prime}+\mathfrak{b}^{\prime} \mathfrak{c}^{\prime}\right) u^{2} v \\
+\frac{1}{2}\left(\mathfrak{b}_{0}^{\prime}+\mathfrak{b}^{\prime 2}\right) u v^{2}+\frac{1}{4 !} B^{40} u^{4}+\cdots,
\end{aligned}
\end{aligned}
$$

$$
\begin{aligned}
& t_{3}=v+\mathfrak{c}^{\prime} u v+\frac{1}{2}\left(\mathfrak{c}_{w}^{\prime}+{\mathfrak{c}^{\prime 2}}^{2}\right) u^{2} v+\frac{1}{2}\left(\mathfrak{c}_{v}^{\prime}+\mathfrak{b}^{\prime}+\mathfrak{b}^{\prime} \mathfrak{c}^{\prime}\right) u v^{2} \\
& +\frac{1}{6} c^{\prime \prime} v^{8}+\frac{1}{4 !} c^{40} u^{4}+\cdots, \\
& t_{4}=1+\frac{1}{2} b u^{2}+b^{\prime} u v+\frac{1}{b}\left(b_{u}-2 c^{\prime} b\right) u^{3}+\frac{1}{2}\left(b_{v}-2 c^{\prime} b^{\prime}\right) u^{2} v \\
& +\frac{1}{2}\left(b_{*}^{\prime}+b^{\prime} b^{\prime}\right) u v^{2}+\frac{1}{b} b^{\prime \prime} v^{2}+\frac{1}{4 !} D^{40} u^{4}+\cdots .
\end{aligned}
$$

Trane. Am. Math. S00. 18 
Let non-homogeneous coördinates be introduced by putting

$$
\zeta=2 \frac{t_{1}}{t_{4}}, \quad \eta=\frac{t_{2}}{t_{4}}, \quad \xi=\frac{t_{3}}{t_{4}},
$$

so that the development of the surface, exact up to terms of the sixth order, takes the form where

$$
\zeta=\xi^{2}+\phi_{3}(\xi, \eta)+\phi_{4}(\xi, \eta)+\phi_{5}(\xi, \eta)+\phi_{6}(\xi, \eta)+\cdots,
$$

$$
\phi_{3}(\xi, \eta)=\zeta_{03} \xi^{3}+\zeta_{12} \eta \xi^{2}+\zeta_{21} \eta^{2} \xi+\zeta_{30} \eta^{3} \text {, etc. }
$$

The explicit expressions for these coefficients $\zeta_{i j}$, which do not vanish identically, are

$$
\begin{aligned}
& \zeta_{12}=-c^{\prime}, \quad \zeta_{03}=-\frac{1}{8} \mathfrak{b}^{\prime}, \quad \zeta_{22}={c^{\prime 2}}^{2}, \\
& \zeta_{18}=-\frac{1}{8}\left(\mathfrak{b}_{4}^{\prime}+3 \mathfrak{c}_{v}^{\prime}-2 \mathfrak{b}^{\prime} \mathfrak{c}^{\prime}\right), \quad \zeta_{04}=\frac{1}{12}\left(\mathfrak{b}^{\prime 2}-\mathfrak{b}_{v}^{\prime}-3 \mathfrak{c}^{\prime \prime}\right), \\
& \zeta_{32}=-{\mathfrak{c}^{\prime 3}}^{3}, \quad \zeta_{23}=\mathfrak{c}^{\prime}\left(\mathfrak{b}_{u}^{\prime}-\mathfrak{b}^{\prime} \mathfrak{c}^{\prime}+3 \mathfrak{c}_{v}^{\prime}\right) \text {, } \\
& \zeta_{14}=\frac{1}{12}\left(16 \mathfrak{b}^{\prime} \mathfrak{c}_{v}^{\prime}+3 \mathfrak{c}^{\prime} \mathfrak{b}_{v}^{\prime}-\mathfrak{b}^{\prime 2} \mathfrak{c}^{\prime}+2 \mathfrak{b}^{\prime} \mathfrak{b}^{\prime}-3 \mathfrak{c}_{v 0}^{\prime}-\mathfrak{b}_{u v}^{\prime}+6 \mathfrak{b}^{\prime} \mathfrak{b}_{u}^{\prime}+9 \mathfrak{c}^{\prime} \mathfrak{c}^{\prime \prime}\right) \text {, } \\
& \zeta_{0 b}=\frac{1}{12}\left(25 \mathfrak{b}^{\prime \prime}+47 \mathfrak{c}_{v}^{\prime \prime}-45 \mathfrak{b}^{\prime} \mathfrak{c}^{\prime \prime}-24 \mathfrak{b}_{v v}^{\prime}+72 \mathfrak{b}^{\prime} \mathfrak{b}_{v}^{\prime}-24 \mathfrak{b}^{\prime 3}\right) \text {, } \\
& \zeta_{42}={\mathfrak{c}^{\prime}}^{4}, \quad \zeta_{83}=\frac{2}{8} \mathfrak{c}^{\prime 2}\left(2 \mathfrak{b}^{\prime} \mathfrak{c}^{\prime}-9 \mathfrak{c}_{v}^{\prime}-3 \mathfrak{b}_{u}^{\prime}\right) \text {, } \\
& \zeta_{24}=\frac{1}{12}\left(-2 \mathfrak{b}^{\prime 2} \mathfrak{c}^{\prime 2}-6 \mathfrak{c}^{\prime 2} \mathfrak{b}_{v}^{\prime}-18 \mathfrak{c}^{\prime \prime} \mathfrak{c}^{\prime 2}-64 \mathfrak{b}^{\prime} \mathfrak{c}^{\prime} \mathfrak{c}_{v}^{\prime}-8 \mathfrak{b}^{\prime} \mathfrak{c}^{\prime} \mathfrak{b}^{\prime}+12 \mathfrak{c}^{\prime} \mathfrak{c}_{v v}^{\prime}\right. \\
& \left.+4 \mathfrak{c}^{\prime} \mathfrak{b}_{u v}^{\prime}-24 \mathfrak{b}_{u}^{\prime} \mathfrak{b}^{\prime} \mathfrak{c}^{\prime}+3 \mathfrak{b}_{u}^{\prime 2}+27 \mathfrak{c}_{v}^{\prime 2}+18 \mathfrak{b}_{u}^{\prime} \mathfrak{c}_{v}^{\prime}\right), \\
& \zeta_{1 b}=\frac{1}{6}\left(6 b_{u}^{\prime \prime}-3 c_{u v}^{\prime \prime}+13 \mathfrak{b}_{u}^{\prime} c^{\prime \prime}+13 \mathfrak{b}^{\prime} \mathfrak{c}_{u}^{\prime \prime}-\mathfrak{b}_{u v 0}^{\prime}+8 \mathfrak{b}^{\prime} \mathfrak{b}_{u v}^{\prime}+13 \mathfrak{b}_{u}^{\prime} \mathfrak{b}_{v}^{\prime}\right. \\
& -23 \mathfrak{b}^{\prime 2} \mathfrak{b}_{u}^{\prime}-619 \mathfrak{c}^{\prime} b^{\prime \prime}-1,178 \mathfrak{c}^{\prime} \mathfrak{c}_{v}^{\prime \prime}+1,138 \mathfrak{b}^{\prime} \mathfrak{c}^{\prime} \mathfrak{c}^{\prime \prime}+599 \mathfrak{c}^{\prime} \mathfrak{b}_{v v}^{\prime}-1,827 \mathfrak{c}^{\prime} \mathfrak{b}^{\prime} \mathfrak{b}_{\text {v }}^{\prime} \\
& \left.+549 \mathfrak{b}^{\prime 3} \mathfrak{c}^{\prime}-50 \mathfrak{b}^{\prime 2} \mathfrak{b}^{\prime}-30 \mathfrak{b}^{\prime} \mathfrak{b}_{v}^{\prime}+45 \mathfrak{c}_{v}^{\prime} \mathfrak{c}^{\prime \prime}+45 \mathfrak{c}_{v}^{\prime} \mathfrak{b}_{v}^{\prime}-75 \mathfrak{c}_{v}^{\prime} \mathfrak{b}^{\prime 2}+15 \mathfrak{b}^{\prime} \mathfrak{c}_{v v}^{\prime}\right) \text {, } \\
& \zeta_{06}=\frac{1}{86} \delta\left(-84 \mathfrak{b}_{v}^{\prime \prime}+84 \mathfrak{b}^{\prime} \mathfrak{b}^{\prime \prime}-3 \mathfrak{c}_{v 0}^{\prime \prime}-28 \mathfrak{b}^{2} \mathfrak{c}^{\prime \prime}+45 \mathfrak{c}^{\prime \prime 2}-\mathfrak{b}_{v 0 v}^{\prime}\right. \\
& \left.+3 \mathfrak{b}_{v}^{\prime 2}+\mathfrak{b}^{\prime 4}+28 \mathfrak{b}_{v}^{\prime} \mathfrak{c}^{\prime \prime}+6 \mathfrak{b}^{\prime} \mathfrak{c}_{v}^{\prime \prime}-4 \mathfrak{b}^{\prime 2} \mathfrak{b}_{v}^{\prime}+4 \mathfrak{b}^{\prime} \mathfrak{b}_{v v}^{\prime}\right) \text {. }
\end{aligned}
$$

Since the surface is developable, the equation

$$
\left(\frac{\partial^{2} \zeta}{\partial \xi \partial \eta}\right)^{2}-\frac{\partial^{2} \zeta}{\partial \xi^{2}} \frac{\partial^{2} \zeta}{\partial \eta^{2}}=0
$$

must be satisfied identically, so that the following relations among the coefficients must hold,

$$
\begin{aligned}
\zeta_{22} & =\zeta_{12}^{2}, \quad \zeta_{23}=3 \zeta_{12}\left(\zeta_{18}-\zeta_{03} \zeta_{12}\right), \quad \zeta_{82}=\zeta_{12}^{3}, \\
4 \zeta_{24} & =12 \zeta_{12}^{2}\left(3 \zeta_{03}^{2}-2 \zeta_{04}\right)+4 \zeta_{12}\left(4 \zeta_{14}-9 \zeta_{03} \zeta_{12}\right)+9 \zeta_{13}^{2}, \\
\zeta_{88} & =6 \zeta_{12}^{2} \zeta_{18}-8 \zeta_{12}^{3} \zeta_{03}, \quad \zeta_{42}=\zeta_{12}^{4} .
\end{aligned}
$$

These equations will be used repeatedly in what follows, without further reference to them. 
Every term in the right member of equation (85) is divisible by $\xi^{2}$, that is, the plane $\zeta=0$ is tangent to the surface at all points of the generator $\xi=\zeta=0$. The most general transformation of coördinates which leaves this plane and this generating line unchanged is

$$
\xi=\lambda \frac{a^{\prime} x+c^{\prime} z}{1+a x+b y+c z}, \quad \eta=\mu \frac{a^{\prime \prime} x+b^{\prime \prime} y+c^{\prime \prime} z}{1+a x+b y+c z},
$$

$$
\zeta=\frac{\nu z}{1+a x+b y+c z},
$$

where $\lambda, \mu, \nu, a, b, \cdots c^{\prime \prime}$, are arbitrary constants, which may be chosen in such a way as to simplify the development into series.*

If, instead of $y$, the linear combination $a^{\prime \prime} x+b^{\prime \prime} y$ is taken, as Darboux has suggested, the transformation (88) may be replaced by the following three. First put

$$
\xi=\frac{x+h z}{1+u_{1}+u_{0} z}, \quad \eta=\frac{y+k z}{1+u_{1}+u_{0} z}, \quad \zeta=\frac{z}{1+u_{1}+u_{0} z},
$$

where $u_{1}$ is a linear function of $x$ and $y$, say,

$$
u_{1}=A x+B y,
$$

and $u_{0}$ is a constant. Follow this transformation by a linear transformation of $x$ and $y$,

$$
x=a^{\prime} x^{\prime}, \quad y=a^{\prime \prime} x^{\prime}+b^{\prime \prime} y^{\prime} ;
$$

and finally by a transformation of the form

$$
\xi=\lambda x, \quad \eta=\mu y, \quad \zeta=\nu z .
$$

If the values of $\xi, \eta, \zeta$, given by equations (89), be substituted into the development (85), it becomes

$$
z=\frac{(x+h z)^{2}}{1+u_{1}+u_{0 . z}}+\frac{\phi_{3}(x+h z, y+k z)}{\left(1+u_{1}+u_{0} z\right)^{2}}+\cdots .
$$

This, of course, must be of the form

$$
z=x^{2}+U_{3}+U_{4}+\cdots,
$$

and, up to terms of the fourth order, the following equation must hold

$$
z=(x+h z)^{2}\left(1-u_{1}-u_{0} z+u_{1}^{2}\right)+\phi_{3}(x, y)\left(1-2 u_{1}\right)+z \Delta \phi_{3}+\phi_{4},
$$

where $\Delta$ represents the operation $h \frac{\partial}{\partial x}+k \frac{\partial}{\partial y}$. Replacing $z$ by the develop-

*The development to terms of the fifth order inclusive is given by Darboux, B ul letin des Sciences mathématiques, 2d series, vol. 4, 1880, p. 356. 
ment (92), and equating the terms of the same degree, we find

$$
\begin{aligned}
& U_{3}=-u_{1} x^{2}+2 h x^{3}+\phi_{3}, \\
& U_{4}=-u_{0} x^{4}+u_{1}^{2} x^{2}-h u_{1} x^{3}+2 h x U_{3}-2 u_{1} \phi_{3}+x^{2} \Delta_{3}+\phi_{4} .
\end{aligned}
$$

The two constants involved in $u_{1}$, may be chosen so as to make $U_{3}$ vanish identically. To accomplish this, it is necessary and sufficient that they satisfy the equations

$$
2 h-A+\zeta_{03}=0, \quad-B+\zeta_{12}=0 .
$$

If these conditions are satisfied the terms of the fourth order become

$$
U_{4}=\left(-u_{0}-A^{2}+\zeta_{04}+3 h \zeta_{03}+k \zeta_{12}\right) x^{4}+\left(\zeta_{13}-2 \zeta_{12} \zeta_{03}\right) x^{8} y .
$$

Let us suppose that $U_{3}$ has been reduced to zero in the way indicated, so that for the new development

$$
\phi_{3}=\phi_{3}^{\prime}=0, \quad \phi_{4}=\phi_{4}^{\prime} .
$$

In order that $U_{8}$ may remain zero for any subsequent transformation of form (89), it is only necessary to have

$$
2 h x-u_{1}=0, \quad \text { that is, } A=2 h, \quad B=0 .
$$

Upon making such a transformation, the new $U_{4}$ becomes

$$
U_{4}=-u_{0} x^{4}+\phi_{4}^{\prime}=\left(\zeta_{04}^{\prime}-u_{0}\right) x^{4}+\left(\zeta_{13}-2 \zeta_{12} \zeta_{03}\right) x^{3} y .
$$

By putting $u_{0}=\zeta_{04}^{\prime}$ it is, therefore, possible to reduce the development to the form

where

$$
z=x^{2}+\Delta_{13} x^{3} y+\phi_{3}(x, y)+\phi_{6}(x, y)+\cdots,
$$

$$
\Delta_{18}=\zeta_{13}-2 \zeta_{12} \zeta_{08}=\frac{1}{3} L,
$$

$L$ being the invariant found in $\$ 8$.

The development may be further simplified by making another transformation of the form (89), viz.,

$$
x=\frac{x^{\prime}+h z^{\prime}}{1+2 h x^{\prime}}, \quad y=\frac{y^{\prime}+k z^{\prime}}{1+2 h x^{\prime}}, \quad z=\frac{z^{\prime}}{1+2 h x^{\prime}},
$$

where we have taken

$$
u_{0}=0, \quad B=0, \quad A=2 h,
$$

so as not to disturb the form of development already established. The new development becomes

$$
\begin{aligned}
& z^{\prime}=\frac{\left(x^{\prime}+h z^{\prime}\right)^{2}}{1+2 h x^{\prime}}+\Delta_{13} \frac{\left(x^{\prime}+h z^{\prime}\right)^{3}\left(y^{\prime}+k z^{\prime}\right)}{\left(1+2 h x^{\prime}\right)^{2}}+\frac{\phi_{b}\left(x^{\prime}+h z^{\prime}, y^{\prime}+k z^{\prime}\right)}{\left(1+2 h x^{\prime}\right)^{4}}+\cdots \\
& =\left(x^{\prime}+h z^{\prime}\right)^{2}\left(1-2 h x^{\prime}+4 h^{2}{x^{\prime}}^{2}-8 h^{3}{x^{\prime}}^{3}+16 h^{4} x^{14}\right) \\
& +\Delta_{18}\left(x^{\prime 3}+3 h x^{\prime 2} z^{\prime}+3 h^{2} x^{\prime}{z^{\prime 2}}^{2}\right)\left(y^{\prime}+k z^{\prime}\right)\left(1+12 h^{2} x^{\prime 2}-4 h x^{\prime}\right) \\
& +\phi_{b}\left(x^{\prime}, y^{\prime}\right)\left(1-8 h x^{\prime}\right)+z^{\prime} \Delta \phi_{b}+\phi_{b}+\cdots \\
& =x^{\prime 2}+\Delta_{18} x^{\prime 8} y^{\prime}+U_{6}+U_{6}+\cdots \text {, }
\end{aligned}
$$


where

$$
\begin{aligned}
U_{6} & =x^{\prime 5}\left(k \Delta_{13}-2 h^{3}\right)+h \Delta_{13} x^{44} y^{\prime}+\phi_{6}\left(x^{\prime}, y^{\prime}\right) \\
& =x^{\prime 5}\left(k \Delta_{13}-2 h^{8}+z_{05}^{\prime}\right)+x^{\prime 4} y^{\prime}\left(h \Delta_{13}+z_{14}^{\prime}\right)
\end{aligned}
$$

The coefficient of $x^{x^{5}}$ may be made equal to zero by putting

$$
k \Delta_{13}-2 h^{8}+z_{05}^{\prime}=0 \text {. }
$$

The term in $x^{\prime 4} y^{\prime}$ also may be reduced to zero, by choice of $h$, provided $\Delta_{13}$ does not vanish identically,that is, whenever the surface is not a cone.

Suppose that the surface is not a cone, and let the terms of the fifth order be removed, as indicated. $U_{B}$ will remain zero under subsequent transformations of the form considered, only provided $h=k=0$, that is, only if the transformation is the identical transfomation. The terms of the sixth order become

and, by putting,

$$
U_{6}=z_{06}^{\prime} x^{60}+z_{15}^{\prime} x^{\prime^{5}} y^{\prime}+\frac{9}{4} \Delta_{13}^{2} x^{\prime 4} y^{\prime 2}
$$

the series reduces to the form

$$
y^{\prime \prime}=\Delta_{13} y^{\prime} \text {, }
$$

$$
z^{\prime}=x^{\prime 2}+x^{\prime 3} y^{\prime \prime}+z_{06}^{\prime} x^{\prime 6}+z_{15}^{\prime} x^{\prime 5} y^{\prime \prime}+\frac{9}{4} x^{\prime 4} y^{\prime \prime 2}+\cdots \text {. }
$$

It will now be convenient to introduce the linear transformation given by equations (90). But, in order to preserve the form of the development just found, it will be necessary to employ a combination of the transformations (89) and (90). Since all of the terms of this development are of even order, it is clear that the most general transformation which need be considered is one of the form

$$
x^{\prime}=\frac{a^{\prime} X}{1+c Z}, \quad y^{\prime \prime}=\frac{a^{\prime \prime} X+b^{\prime \prime} Y}{1+c Z}, \quad z^{\prime}=\frac{Z}{1+c Z} .
$$

When such a transformation is made, the development becomes

$$
\begin{aligned}
Z=a^{\prime 2} X^{2}(1 & \left.-c Z+c^{2} Z^{2}\right)+a^{\prime 8} X^{3}(1-c Z)^{3}\left(a^{\prime \prime} X+b^{\prime \prime} Y\right)+z_{06}^{\prime} a^{\circ} X^{6} \\
& +z_{15}^{\prime} a^{\prime 5} X^{6}\left(a^{\prime \prime} X+b^{\prime \prime} Y\right)+\frac{9}{a^{\prime 4}} X^{4}\left(a^{\prime \prime} X+b^{\prime \prime} Y\right)^{2}+\cdots
\end{aligned}
$$

In order that this may again assume the form

it is necessary to have

$$
Z=X^{2}+X^{8} Y+U_{6}+\cdots,
$$

$$
a^{\prime 2}=1, \quad-a^{\prime 2} c+a^{\prime 8}\left(a^{\prime \prime}=0, \quad b^{\prime \prime} a^{\prime 3}=1 .\right.
$$

Assuming these equations to be satisfied, the terms of the sixth order become

$$
U_{6}=\left(Z_{06}-2 c^{2}\right) X^{6}+\left(Z_{16}-4 c\right) X^{5} Y+\frac{9}{2} X^{4} Y^{2},
$$

so that it is possible to reduce either of the first two terms to zero by a proper 
choice of the constant $c$. Let the term in $X^{5} Y$ be removed, so that the series will assume the form

$$
Z=X^{2}+X^{3} Y+\Delta_{06} X^{6}+\frac{9}{4} X^{4} Y^{2}+\cdots .
$$

By applying a transformation of the form (91), viz:

$$
X^{\prime}=\lambda X, \quad Y^{\prime}=\mu Y, \quad Z^{\prime}=\nu Z,
$$

this may be made finally to take the form

$$
Z^{\prime}=X^{\prime 2}+X^{\prime 3} Y^{\prime}+k X^{\prime 6}+\frac{9}{4} X^{\prime 4} Y^{\prime 2}+\cdots,
$$

where $k$ is unity or zero, according as $\Delta_{06}$ does not, or does, vanish. In fact, to accomplish this, it is only necessary to put $\nu=\lambda^{2}=\lambda^{3} \mu$, and, if $\Delta_{06} \neq 0$, $\lambda=\sqrt[4]{\Delta_{06}}$. If $\Delta_{06}=0, \lambda$ may be left arbitrary here, and determined subsequently so as to simplify the terms of orders higher than the sixth.

In obtaining the terms of the fifth and sixth orders in this development, we have assumed that the invariant $\Delta_{13}$ does not vanish, that is, that the surface is not a cone. If the surface is a cone, $\Delta_{13}$ is necessarily zero, and these terms cannot be reduced as in the case just treated. In fact, it can be shown that, in general, the term in $X^{5}$ must remain.

The results may be summarized as follows: For every regular point of a developable surface, a tetrahedron of reference may be so chosen that, in the vicinity of this point, the equation of the surface may be expanded in the form

$$
\begin{aligned}
z=x^{2}+k_{13} x^{3} y+\left(1-k_{13}\right) & k_{05}\left(1-k_{06}\right) x^{5}+\left(1-k_{05}\right) k_{06} x^{6} \\
& +\left(1-k_{13}\right) k_{15} x^{5} y+\frac{9}{4} k_{13} x^{4} y^{2}+R_{6},
\end{aligned}
$$

where each of the quantities $k_{i j}$ may have either the value 0 or 1 according as a corresponding invariant $\Delta_{i j}$ vanishes or does not vanish, and where $R_{6}$ is the remainder after terms of the sixth order.

In reducing the development (85) to the various forms (94), we have purposely avoided the complications which would be involved in preserving the explicit expressions for the coefficients in the several intermediate developments. When these expressions are preserved, the invariants $\Delta_{i j}$ appear in the process of reduction. They are

$$
\begin{aligned}
& \Delta_{13}=\zeta_{13}-2 \zeta_{12} \zeta_{03}, \quad \Delta_{05}=\zeta_{05}+2 \zeta_{03}^{3}-3 \zeta_{03} \zeta_{04}, \\
& \Delta_{16}=\zeta_{15}+8 \zeta_{12} \zeta_{03}^{3}-12 \zeta_{12} \zeta_{03} \zeta_{04}+6 \zeta_{12} \Delta_{05} \text {, } \\
& \Delta_{13}^{4} \Delta_{06}=\Delta_{13}^{4}\left(D_{06}-4 \zeta_{03} \Delta_{05}\right)-\Delta_{13}^{3} \Delta_{05} D_{14}-\left[D_{14}^{2}-2 \Delta_{13}^{2} \zeta_{03}^{2}\right. \\
& \left.-\Delta_{13}\left(\Delta_{15}-10 \zeta_{12} \Delta_{05}-4 \zeta_{04} \Delta_{13}\right)\right]^{2} \text {, }
\end{aligned}
$$

where

$$
\begin{aligned}
& D_{14}=\zeta_{14}+6 \zeta_{12} \zeta_{03}^{2}-3 \zeta_{12} \zeta_{04}-3 \zeta_{13} \zeta_{03}, \\
& D_{08}=\zeta_{06}+3 \zeta_{03}^{4}-2 \zeta_{03}^{2} \zeta_{04}-2 \zeta_{04}^{2} .
\end{aligned}
$$


Consider that form of the development (94) for which $\Delta_{18}$ is different from zero, that is,

$$
z=x^{2}+x^{3} y+k x^{6}+\frac{9}{4} x^{4} y^{2}+R_{6},
$$

where $k=1$, or 0 , according as $\Delta_{06}$ is different from, or equal to zero.

Let the transformation of form (88) which will project the surface (94a) into the surface (85) be

$x=\frac{\lambda_{1}(\xi+\lambda \zeta)}{1+\alpha \xi+\beta \eta+\gamma \zeta}, \quad y=\frac{\mu_{1}(\rho \xi+\eta+\mu \zeta)}{1+\alpha \xi+\beta \eta+\gamma \zeta}, \quad z=\frac{\nu_{1} \zeta}{1+\alpha \xi+\beta \eta+\gamma \zeta}$.

If homogeneous coördinates

$$
x_{1}=z, \quad x_{2}=y, \quad x_{3}=x, \quad x_{4}=1,
$$

be introduced, the relations between these coördinates and those of the original system are defined by the equations

$$
\begin{aligned}
& \omega t_{1}=\frac{1}{2 \nu_{1}} x_{1}, \\
& \omega t_{2}=\frac{1}{\nu_{1}}(\lambda \rho-\mu) x_{1}+\frac{1}{\mu_{1}} x_{2}-\frac{\rho}{\lambda_{1}} x_{3}, \\
& \omega t_{3}=-\frac{\lambda}{\nu} x_{1}+\frac{1}{\lambda_{1}} x_{3}, \\
& \omega t_{4}=\frac{1}{\nu_{1}}[\alpha \lambda-\beta(\lambda \rho-\mu)-\gamma] x_{1}-\frac{\beta}{\mu_{1}} x_{2}+\frac{\beta \rho-\alpha}{\lambda_{1}} x_{3}+x_{4},
\end{aligned}
$$

where

$$
\begin{gathered}
\Delta_{18} \alpha=-2 D_{14}-\zeta_{08} \Delta_{18}, \quad \beta=-\zeta_{12}, \quad \Delta_{18} \lambda=-D_{14}, \\
\Delta_{13}^{2} \rho=-10 \lambda^{2} \Delta_{18}+2 \lambda\left(5 \zeta_{08} \Delta_{18}-4 D_{14}\right)-2 \Delta_{18}\left(5 \zeta_{03}^{2}+4 \zeta_{04}\right) \\
+2 \zeta_{08}\left(D_{14}+3 \zeta_{03} \Delta_{18}\right)-8 \zeta_{12} \Delta_{05}+2 \Delta_{16}-12 \zeta_{12} \Delta_{05}, \\
\Delta_{18}(\lambda \rho-\mu)+\Delta_{08}=0, \quad \gamma=\Delta_{13} \rho+\lambda^{2}+\zeta_{03}^{2}-\zeta_{04}, \quad \lambda_{1} \mu_{1}=\Delta_{18}, \\
\nu_{1}=\lambda_{1}^{2}
\end{gathered}
$$

and where $\lambda_{1}=\sqrt[4]{\Delta_{06}}$ if $\Delta_{06} \neq 0$. If $\Delta_{06}=0, \lambda_{1}$ is to be left arbitrary until the form of the remainder $R_{6}$ is determined.

The vertex $(0,0,0,1)$ of the canonical tetrahedron is the point $y$. Let the coördinates which represent the other vertices $(1,0,0,0),(0,1,0,0)$ $(0,0,1,0)$ be denoted by $\mathfrak{q}^{\prime}, \mathfrak{r}^{\prime}$, and $\boldsymbol{s}^{\prime}$ respectively. Since $\mathfrak{r}^{\prime}=\mathfrak{D}$, this vertex of the canonical tetrahedron lies on the cuspidal edge of the integral surface.

\section{\$11. The canonical quartic scroll.}

Let a developable surface $S$, not a cone, be referred to the canonical tetrahedron (defined by equation (96)), which belongs to one of its points $y$ and consider the quartic surface

$$
z=x^{2}+x^{3} y,
$$


which shall be called the canonical quartic of the point. The equation of the latter in the homogeneous coördinates (95) is

$$
x_{1} x_{4}^{3}-x_{3}^{2} x_{4}^{2}-x_{3}^{3} x_{2}=0 .
$$

The equations of the first, second and third polars of a point $\left(y_{1}, y_{2}, y_{3}, y_{4}\right)$ with respect to the surface are respectively

$$
\begin{aligned}
& y_{4}^{3} x_{1}-y_{3}^{3} x_{2}-\left(3 y_{3}^{2} y_{2}+2 y_{4}^{2} y_{3}\right) x_{3}+\left(3 y_{4}^{2} y_{1}-2 y_{4} y_{3}^{2}\right) x_{4}=0,=0 \\
& \text { (99) }-\left(6 y_{3} y_{2}+2 y_{4}^{2}\right) x_{3}^{2}+\left(6 y_{4} y_{1}-2 y_{3}^{2}\right) x_{4}^{2}-3 y_{3}^{2} x_{3} x_{2}-4 y_{4} y_{3} x_{4} x_{8}+3 y_{4}^{2} x_{4} x_{1}=0, \\
& 3 y_{1} x_{4}^{3}-2 y_{3} x_{4}^{2} x_{3}+3 y_{4} x_{4}^{2} x_{1}-2 y_{4} x_{4} x_{3}^{2}-3 y_{2} x_{3}^{3}-3 y_{3} x_{3}^{3} x_{2}=0 .
\end{aligned}
$$

The only singular points of the surface $Q$, therefore, lie on the line $x_{3}=x_{4}=0$, and every point of this line $t$ is a triple point on the surface. Thus the surface is a scroll. The tangent planes to the surface $Q$ along the triple line $t$ are given by the equation

$$
y_{1} x_{4}^{3}-y_{2} x_{3}^{3}=0 \text {. }
$$

The three tangent planes coincide for $y_{1}=0$, or $y_{2}=0$, so that $q^{\prime}=(1,0,0,0)$ and $\mathbb{Q}=(0,1,0,0)$ may be called triple pinch points on the quartic scroll $Q . *$

In order to complete the determination of the canonical tetrahedron, we must find out how the scroll $Q$ is determined by the properties of the surface $S$. It is easily seen that the most general quartic scroll which has the line $x_{3}=x_{4}=0$ for a triple line and a pair of triple pinch points on that line, is given by the equation

$$
\left(a x_{4}+h x_{3}\right)^{3} x_{1}+\left(b x_{3}+k x_{4}\right)^{3} x_{2}+c x_{4}^{4}+d x_{4}^{3} x_{3}+e x_{4}^{2} x_{3}^{2}+f x_{4} x_{3}^{3}+g x_{3}^{4}=0 .
$$

If this surface passes through the point $y=(0,0,0,1)$, and has the tangent plane $\tau$, or $x_{1}=0$, in common with the surface $S$, we must have

$$
c=k=d=0 \text {. }
$$

Let the triple line, instead of being $x_{3}=x_{4}=0$, be the arbitrary line (not passing through $y \dagger$ )

$$
\begin{aligned}
& x_{1}+l x_{1}+m x_{2}=0, \\
& x_{3}+p x_{1}+q x_{2}=0,
\end{aligned}
$$

and let the pinch points be arbitrary points of this line, viz., the points of intersection of this line with the planes

$$
x_{1}+\lambda x_{2}=0, \quad x_{2}+\mu x_{1}=0 .
$$

* This species of scrolls is described by Carusr. See Collected Mathematical Papers, Vol. VI, p. 312.

† This will be seen to imply only that $y$ is not a point of the cuspidal edge of $S$. 
The equation of the most general quartic scroll having a triple line with two triple pinch points and touching the surface $S$ at the point $y$ will then be obtained by putting,

$$
x_{1}+\lambda x_{2}, \quad x_{2}+\mu x_{1}, \quad x_{3}+p x_{1}+q x_{2}, \quad x_{1}+l x_{1}+m x_{2},
$$

in place of $x_{1}, x_{2}, x_{3}, x_{4}$, respectively, in

$$
\phi\left(x_{1}, x_{2}, x_{3}, x_{4}\right)=\left(a x_{4}+h x_{3}\right)^{3} x_{1}+b x_{3}^{3} x_{2}+e x_{4}^{2} x_{3}^{2}+f x_{1} x_{3}^{3}
$$

that is, in non-homogeneous coördinates

$$
+g x_{3}^{4}=0 \text {, }
$$

$$
\phi(z+\lambda y, \quad y+\mu z, \quad x+p z+q y, \quad 1+l z+m y)=0 .
$$

We shall assume that this quartic scroll has contact of the fifth order with $S$ at the point $y$. Then the development

$$
z=x^{2}+x^{3} y+k x^{6}+\frac{9}{4} x^{4} y^{2}+\cdots,
$$

where $k=0$, or 1 , according as $\Delta_{00}$ does, or does not, vanish, must satisfy equation (102) up to terms of the fifth order inclusive. The conditions obtained in this way give, if we put $a=1$,

$$
\begin{gathered}
l=q=m=0, \quad e=b=-1, \quad f=-3 p, \quad h=\frac{5}{8} p, \\
g=-l-\frac{10}{8} p^{2}, \quad \mu=-\frac{28}{2} p^{3}-p l .
\end{gathered}
$$

Thus, to every regular point $y$ of the surfaces $S$ there belongs a family of $\infty^{2}$ quartic scrolls, having contact of the fifth order with $S$ at $y$, and carrying a pair of triple pinch points on a triple line. The equation of the most general quartic scroll of this kind is

$$
\begin{aligned}
& {\left[x_{4}+l x_{1}+h\left(x_{3}+p x_{1}\right)\right]^{3} x_{1}-\left(x_{3}+p x_{1}\right)^{3}\left(x_{2}+\mu x_{1}\right)} \\
& -\left(x_{3}+p x_{1}\right)^{2}\left(x_{4}+l x_{1}\right)^{2}+f\left(x_{4}+l x_{1}\right)\left(x_{3}+p x_{1}\right)^{3} \\
& +g\left(x_{3}+p x_{1}\right)^{4}=0,
\end{aligned}
$$

where $f, g, h, \mu$, are the functions of $p$ and $l$ given above.

Consider a surface $Q$ of this family. Every plane which contains the point $y$ will intersect the surfaces $Q$ and $S$ in plane curves which have contact of at least the fifth order with each other at $y$. Among these planes there are some which intersect $Q$ and $S$ in a pair of curves having contact of at least the sixth order with each other at $y$. These planes may be found by substituting the development (94a) for $S$ into equation (103) for the scroll $Q$, and equating to zero the terms of the sixth order which do not vanish identically. We obtain in this way a sextic homogeneous equation in $x$ and $y$, which may be reduced to

$$
x^{4}\left(4 S x^{2}+4 R x y+27 y^{2}\right)=0,
$$


where

$$
R=12 l+13 p^{2}, \quad S=3 k+6 l^{2}+31 p^{2} l+67 p^{4} ;
$$

or in homogeneous coördinates,

$$
x_{3}^{4}\left(4 S x_{3}^{2}+4 R x_{3} x_{2}+27 x_{2}^{2}\right)=0 .
$$

This equation determines six tangents of the surface, that is, the generator $g$ counted four times, and two lines $t_{1}, t_{2}$, which we shall call the tangents of sixth order contact between $Q$ and $S$, because every plane passing through such a line intersects $Q$ and $S$ in a pair of curves having contact of at least the sixth order with each other at $y$.

The tangent plane $\tau$, or $x_{1}=0$, to the surface $S$ at the point $y$, intersects the quartic $Q$ in a quartic curve consisting of the generator $g$ counted twice and a conic $K$, whose equation is

$$
g x_{3}^{2}+f x_{3} x_{4}-x_{4}^{2}-x_{3} x_{2}=0 .
$$

The polar of the point $y$ with respect to the conic $K$ is

$$
2 x_{4}+p x_{3}=0 \text {. }
$$

This line intersects $K$ in two points $K_{1}, K_{2}$, whose coördinates are respectively,

$$
x_{1}=x_{3}=0, \quad x_{1}=4 x_{2}-R x_{3}=0 .
$$

The tangent $t$ to $K$ at the point $K_{2}$ has for its earlation

$$
4 x_{2}-R x_{3}=0 \text {, }
$$

while the tangent of $K$ at $K_{1}$ coincides with $g$.

Consider the four lines $g, t, t_{1}, t_{2}$. They all lie in the plane $\tau$ and pass through the point $y$. The lines $t_{1}$ and $t_{2}$ will be harmonic conjugates with respect to $g$ and $t$ if, and only if, $R=0$. But, when $R=0, l=-\frac{18}{12} p^{2}$, so that there remains a single infinity of quartic scrolls $Q_{h}$ whose tangents of sixth order contact with $S$ divide $g$ and $t$ harmonically. For every surface of this family the line coincides with the edge $x_{1}=x_{2}=0$ of the canonical tetrahedron.

Consider one of the scrolls $Q_{h}$ of this family. The plane $x_{2}+\mu x_{1}=0$, determined by the pinch point $q^{\prime}$ and the line $t$, intersects $Q_{h}$ in a quartic curve $C_{4}$ whose equations are

$$
x_{2}+\mu x_{1}=0 \text {, }
$$

$$
\begin{array}{r}
{\left[x_{4}+l x_{1}+h\left(x_{3}+p x_{1}\right)\right]^{3} x_{1}-\left(x_{3}+p x_{1}\right)^{2}\left(x_{4}+l x_{1}\right)^{2}+f\left(x_{4}+l x_{1}\right)} \\
\left(x_{3}+p x_{1}\right)^{3}+g\left(x_{3}+p x_{1}\right)^{4}=0,
\end{array}
$$

where $f, g, h, \mu$ are all constant multiples of positive powers of $p$. If we refer 
the curve $C_{4}$ to a set of non-homogeneous coördinates,

$$
\rho Z=x, \quad \rho Y=\mu x_{1}+x_{2}, \quad \rho X=p x_{1}+x_{3}, \quad \rho=l x_{1}+x_{4},
$$

its equations become

$$
Y=0, \quad(1+h X)^{3} Z-X^{2}+f X^{3}+g X^{4}=0 .
$$

The necessary and sufficient condition that the left member of the latter equation can be factored into the form

$$
(a+b Z+c X)(d+e X)\left(j X^{2}+k X Z+m X+n Z+q\right),
$$

or that the curve degenerates into a pair of lines and a conic, is that $g=0$. But when $g=0, p=\mu=h=f=0$, and equation (103) becomes identical with that of the canonical quartic scroll (98).

The canonical quartic scroll $Q$ of a point $y$ is, therefore, completely characterized by the following properties.

1. It has a triple line with two triple pinch points, $\mathfrak{Q}, \mathfrak{q}^{\prime}$, the first of which lies at the intersection of the cuspidal edge of the given surface $S$ with the generator $g$ passing through the point $y$.

2. It has the contact of the fifth order with $S$ at $y$.

3. The tangent plane $\tau$ to $S$ at $y$ intersects $Q$ in the generator counted twice and a conic $K$ tangent to $g$. The two tangents $t_{1}, t_{2}$, (distinct from $g$ ) of sixth order contact between $Q$ and $S$ at $y$, divide the two tangents $g$ and $t$, drawn to $K$ from $y$, harmonically.

4. A plane through $t$ and $\mathfrak{q}^{\prime}$ cuts $Q$ in a curve $C_{4}$ which degenerates into a pair of lines and a conic.

For the canonical quartic scroll, given by equation (98), the point $K_{2}$ coincides with the point $\mathfrak{z}^{\prime}$. Thus all of the vertices, $y, \mathfrak{q}^{\prime}, \mathfrak{r}^{\prime}, \mathfrak{s}^{\prime}$, of the canonical tetrahedron have now been determined geometrically. In order to complete the geometrical determination of the system of coördinates which leads to the canonical development, it remains to find the unit point of the system. This may be done as follows.

Consider the two planes determined by the pinch point $\mathfrak{q}^{\prime}$ and the two lines $t_{1}, t_{2}$, whose equations are, by (104),

$$
4 k x_{3}^{2}+9 x_{2}^{2}=0 .
$$

By considering a special case, say that in which $\mathfrak{b}^{\prime}=0$, it can easily be shown that $\Delta_{06}$, and hence $k$, is, in general, different from zero. We shall treat only that case for which $\Delta_{06} \neq 0$. We may then assume $k=1$, so that the equations of the two planes are separately

$$
2 x_{3}+3 i x_{2}=0, \quad 2 x_{3}-3 i x_{2}=0 .
$$


They intersect the surface $Q$ in two quartic curves $C^{\prime}, C^{\prime \prime}$, whose equations are respectively.

$$
x_{1} x_{4}^{3}+\frac{2}{3} i x_{3}^{4}-x_{3}^{2} x_{4}^{2}=0, \quad x_{1} x_{4}^{3}-\frac{2}{3} i x_{3}^{4}-x_{3}^{2} x_{4}^{2}=0 .
$$

The Hessians $H^{\prime}, H^{\prime \prime}$, of $C^{\prime}$ and $C^{\prime \prime}$, are respectively

$$
x_{4}^{4}\left(4 i x_{3}^{2}-x_{4}^{2}\right)=0, \quad x_{4}^{4}\left(4 i x_{3}^{2}+x_{4}^{2}\right)=0 .
$$

Twenty-two of the twenty-four intersections of the curves $C^{\prime}$ and $C^{\prime \prime}$ coincide with the pinch point $\mathfrak{q}^{\prime}$. The other two intersections are the points of inflection

$$
A\left(-\frac{5}{12} \omega^{3}, \frac{2}{3} \omega^{2}, 1,2 \omega\right) ; \quad B\left(\frac{5}{12} \omega^{3}, \frac{2}{3} \omega^{2}, 1,-2 \omega\right),
$$

where $\omega$ is a fourth root of -1, viz., $\frac{1}{2} \sqrt{2}+i \frac{1}{2} \overline{2}$.

The curves $C^{\prime \prime}$ and $H^{\prime \prime}$ intersect in $q^{\prime}$ counted twenty-two times, and in the points of inflection

$$
C\left(-\frac{5}{12} \omega,-\frac{3}{3} \omega^{2}, 1,2 \omega^{3}\right), \quad D\left(\frac{6}{12} \omega,-\frac{3}{3} \omega^{2}, 1,-2 \omega^{3}\right) .
$$

The four points $A, B, C, D$, determine a non-degenerate tetrahedron. If the four fourth roots of -1 are interchanged, these points are permuted according to the four group

$$
1, \quad A B \cdot C D, \quad B C \cdot D A, \quad A C \cdot B D .
$$

These results may be stated as follows. The vertices of the canonical tetrahedron are the given point $y$, the two triple pinch points $\mathfrak{q}^{\prime}, \mathbb{D}$ of the canonical quartic scroll $Q$, and the point of tangency $\vec{b}^{\prime}$, of a tangent from $y$ to the conic $K$, which the tangent plane at $y$ to the surface $S$ cuts out of $Q$. The system of coordinates which gives rise to the canonical development refers to this tetrahedron. If the invariant $\Delta_{06}$ does not vanish identically, the unit point is to be taken in such $a$ way that the points of inflection, $A, B, C, D$, described above, have the coördinates indicated. Since these points may be permuted in four different ways, without altering the system of coördinates, the canonical development may be made in four different ways. For this reason its expression contains the fourth root of a rational invariant.

When $\Delta_{06}=0, k=0$; and the lines $t_{1}, t_{2}$ coincide with $t$. In this case it would be necessary to consider terms of higher order than the sixth in order to determine the unit point of the canonical system of coördinates.

University of ILInNoIs, Urbana, Iux., March 11, 1912. 